MATHEMATICS OF COMPUTATION

Volume 80, Number 273, January 2011, Pages 165-203

S $0025-5718(10) 02368-9$

Article electronically published on April 23, 2010

\title{
SEMIGROUP STABILITY OF FINITE DIFFERENCE SCHEMES FOR MULTIDIMENSIONAL HYPERBOLIC INITIAL-BOUNDARY VALUE PROBLEMS
}

\author{
JEAN-FRANÇOIS COULOMBEL AND ANTOINE GLORIA
}

\begin{abstract}
We develop a simple energy method for proving the stability of finite difference schemes for multidimensional hyperbolic initial-boundary value problems. In particular, we extend to several space dimensions and to variable coefficients a crucial stability result by Goldberg and Tadmor for Dirichlet boundary conditions. This allows us to give some conditions on the discretized operator that ensure that stability estimates for zero initial data imply a semigroup stability estimate for general initial data. We apply this criterion to several numerical schemes in two space dimensions.
\end{abstract}

\section{INTRODUCTION}

The aim of this article is to prove stability estimates for finite difference discretizations of hyperbolic initial-boundary value problems. A general theory to derive such estimates has been developed in [4] for one-dimensional problems and later extended in [11] to multidimensional problems. The analysis for the discretized equations is similar to the theory in 8 for the continuous problem, namely for hyperbolic systems of partial differential equations. It relies on the so-called normal mode analysis. Due to the fact that the method uses a Laplace transform in time, all stability estimates in 4, 8, 11 are restricted to zero initial data. The natural following step in the analysis is then to show that if a problem is stable for zero initial data, then it is also stable for non-zero initial data. Making the space of "suitable" initial data precise is part of the question.

For hyperbolic systems of partial differential equations, this question was solved in [12. The suitable space of initial data is $L^{2}$ as can be expected from the theory of dissipative boundary conditions. We refer to [1, Chapter 4] for a complete description of the results. For discretized problems with constant coefficients, this question was solved in one space dimension in [17] where the author proves a semigroup stability estimate for non-zero initial data. More precisely, the argument in [17. reduces the semigroup stability estimate to the verification of stability for Dirichlet boundary conditions only. The crucial point in 17 is therefore to find a framework in which one can check stability for Dirichlet boundary conditions. For one-dimensional problems, the latter property is a consequence of [2, Lemma 2.3] (see also [17, Lemma 3.2]), which we shall refer to as the Goldberg-Tadmor Lemma.

Received by the editor May 13, 2009 and, in revised form, August 13, 2009; October 1, 2009. 2010 Mathematics Subject Classification. Primary 65M12, Secondary 65M06, 35L50.

The research of the first author was supported by the Agence Nationale de la Recherche, contract ANR-08-JCJC-0132-01.

(C)2010 American Mathematical Society Reverts to public domain 28 years from publication 
This result completes the proof in [17] of the semigroup stability estimate. The author of [17] also notes at the end of his work that his strategy "can be generalized to multidimensional hyperbolic equations with symmetric coefficient matrices". To complete this program, the crucial step is to extend the Goldberg-Tadmor Lemma to multidimensional systems. Such an extension is, however, not straightforward. Indeed, the proof of the Goldberg-Tadmor Lemma for one-dimensional systems relies on an explicit computation of generalized eigenspaces for companion matrices; see [2, eq. (4.6)]. In the case of multidimensional systems, one now faces block companion matrices where each block is itself a square matrix. To adapt the GoldbergTadmor proof, one therefore needs to compute generalized eigenspaces for block companion matrices. Unfortunately, the explicit representation that is known for companion matrices is no longer true for block companion matrices. An alternative approach for proving the Goldberg-Tadmor Lemma is provided in [7, Lemma 8]; see also [6]. The argument of [7] shows the non-existence of unstable eigenvalues for Dirichlet boundary conditions. Yet it does not give information about the existence of generalized eigenvalues 1 . Consequently, it seems that no existing theory yields the Goldberg-Tadmor Lemma for multidimensional systems. Hence the semigroup stability estimate in $[17$ is, so far, restricted to one-dimensional systems or to scalar equations.

The aim of this article is to extend the Goldberg-Tadmor Lemma to several space dimensions and to prove semigroup estimates for $\ell^{2}$ initial data. We first develop a simple energy method with which we recover the Goldberg-Tadmor Lemma and the stability results of [17] in one space dimension and constant coefficients. The crucial novelty of our approach is that it is flexible enough to handle discretized multidimensional systems and variable coefficients as well. Unlike the original proof in 2, our new proof of the Goldberg-Tadmor Lemma covers directly the case of non-zero initial data in $\ell^{2}$, which allows us to bypass some of the arguments of [17] where another set of auxiliary boundary conditions was considered. In particular, our whole approach is self-contained, and we nowhere make use of the elaborate GKS theory [4].

The article is organized as follows. One-dimensional problems with constant coefficients are considered in Section 2 . The results are extended to multidimensional systems with constant coefficients in Section 3. the proof of which can be made elementary by using partial Fourier transform. To deal with variable coefficients, this strategy is no longer applicable. In Section 4 we generalize our results to the case of variable coefficients by combining the approach of Section 2 with pseudo-difference calculus. Then in Section 5, we comment on our results and give examples of discretizations to which they apply.

Notation. Throughout this paper, we let $\mathcal{M}_{d, D}(\mathbb{K})$ denote the set of $d \times D$ matrices with entries in $\mathbb{K}=\mathbb{R}$ or $\mathbb{C}$, and we use the notation $\mathcal{M}_{D}(\mathbb{K})$ when $d=D$. If $M \in \mathcal{M}_{D}(\mathbb{C}), \operatorname{sp}(M)$ denotes the spectrum of $M, \rho(M)$ denotes the spectral radius of $M$, while $M^{*}$ denotes the conjugate transpose of $M$. We let $I$ denote the identity matrix, without mentioning the dimension. The norm of a vector $x \in \mathbb{C}^{D}$ is $|x|:=\left(x^{*} x\right)^{1 / 2}$. The corresponding norm for matrices in $\mathcal{M}_{D}(\mathbb{C})$ is also denoted $|\cdot|$. Eventually, we let $\ell^{2}$ denote the set of square integrable sequences, without

\footnotetext{
${ }^{1}$ According to the theory in 4], stability is equivalent to the non-existence of unstable eigenvalues and generalized eigenvalues.
} 
mentioning the indices of the sequences (sequences may be valued in $\mathbb{C}^{d}$ for some integer $d$ ).

The letter $C$ denotes a constant that may vary from line to line or within the same line. The dependence of the constants on the various parameters is made precise throughout the text.

\section{ONE-DIMENSIONAL PROBLEMS}

For one-dimensional problems, we introduce the following notation for norms on $\ell^{2}(\mathbb{Z})$. Let $\Delta x>0$ be a space step. For all integers $m_{1} \leq m_{2}$, we set

$$
\|u\|_{m_{1}, m_{2}}^{2}:=\Delta x \sum_{j=m_{1}}^{m_{2}}\left|u_{j}\right|^{2}
$$

to denote the $\ell^{2}$-norm on the interval $\left[m_{1}, m_{2}\right]\left(m_{1}\right.$ may equal $-\infty$ and $m_{2}$ may equal $+\infty)$. The corresponding scalar product is denoted by $(\cdot, \cdot)_{m_{1}, m_{2}}$.

2.1. Main result in one space dimension. We consider a hyperbolic initialboundary value problem in one space dimension:

$$
\begin{cases}\partial_{t} u+A \partial_{x} u=F(t, x), & (t, x) \in \mathbb{R}^{+} \times \mathbb{R}^{+}, \\ B u(t, 0)=g(t), & t \in \mathbb{R}^{+}, \\ u(0, x)=f(x), & x \in \mathbb{R}^{+},\end{cases}
$$

where $A \in \mathcal{M}_{D}(\mathbb{R})$ is diagonalizable with real eigenvalues, and $B \in \mathcal{M}_{D_{+}, D}(\mathbb{R})$ with $D_{+}$the number of positive eigenvalues of $A$ (counted with their multiplicity). We assume that the boundary is non-characteristic; that is, $0 \notin \operatorname{sp}(A)$. Problem (11) is well posed in any suitable sense if and only if

$$
\mathbb{R}^{D}=\operatorname{Ker} B \oplus E_{+}(A)
$$

where $E_{+}(A)$ is the unstable eigenspace of $A$ (associated with positive eigenvalues of $A$ ). In that case, the solution $u$ to (1) belongs to $\mathcal{C}\left(\mathbb{R}^{+} ; L^{2}\left(\mathbb{R}^{+}\right)\right)$and its trace on $\{x=0\}$ is well defined and belongs to $\mathrm{e}^{\gamma t} L^{2}\left(\mathbb{R}^{+}\right)$for all $\gamma>0$. Moreover, for every parameter $\gamma>0, u$ satisfies the energy estimate

$$
\begin{aligned}
& \sup _{t \geq 0} \mathrm{e}^{-2 \gamma t}\|u(t, \cdot)\|_{L^{2}\left(\mathbb{R}^{+}\right)}^{2} \\
& \quad+\gamma \int_{0}^{+\infty} \mathrm{e}^{-2 \gamma t}\|u(t, \cdot)\|_{L^{2}\left(\mathbb{R}^{+}\right)}^{2} \mathrm{~d} t+\int_{0}^{+\infty} \mathrm{e}^{-2 \gamma t}|u(t, 0)|^{2} \mathrm{~d} t \\
& \leq C\left(\|f\|_{L^{2}\left(\mathbb{R}^{+}\right)}^{2}+\frac{1}{\gamma} \int_{0}^{+\infty} \mathrm{e}^{-2 \gamma t}\|F(t, \cdot)\|_{L^{2}\left(\mathbb{R}^{+}\right)}^{2} \mathrm{~d} t+\int_{0}^{+\infty} \mathrm{e}^{-2 \gamma t}|g(t)|^{2} \mathrm{~d} t\right),
\end{aligned}
$$

where the constant $C$ is independent of $\gamma, f, F, g$. The estimate (2) can be localized on any finite time interval $[0, T]$ because the solutions to (11) satisfy a causality principle ("future does not affect the past").

We now introduce the finite difference approximation of (11). Let $\Delta x, \Delta t>0$ denote the space and time steps, where the ratio $\lambda:=\Delta t / \Delta x$ is a fixed positive constant, and let $p, q, r$ be some integers. The solution $u$ to (11) is approximated by a sequence $\left(U_{j}^{n}\right)$ defined for $n \in \mathbb{N}$, and $j \in 1-r+\mathbb{N}$. For $j=1-r, \ldots, 0, U_{j}^{n}$ approximates the trace $u(n \Delta t, 0)$ on the boundary $\{x=0\}$, and possibly the trace of normal derivatives. The boundary meshes $[j \Delta x,(j+1) \Delta x[, j=1-r, \ldots, 0$, shrink to $\{0\}$ as $\Delta x$ tends to 0 . Hence the "formal" limit problem as $\Delta x$ tends to 
0 is set on the half-line $\mathbb{R}^{+}$. We consider one-step finite difference approximations of (11) that read2

$$
\begin{cases}U_{j}^{n+1}=Q U_{j}^{n}+\Delta t F_{j}^{n}, & j \geq 1, \quad n \geq 0, \\ U_{j}^{n+1}=B_{j,-1} U_{1}^{n+1}+B_{j, 0} U_{1}^{n}+g_{j}^{n+1}, & j=1-r, \ldots, 0, \quad n \geq 0, \\ U_{j}^{0}=f_{j}, & j \geq 1-r,\end{cases}
$$

where the operators $Q, B_{j,-1}, B_{j, 0}$ are given by

$$
Q:=\sum_{\ell=-r}^{p} A_{\ell} T^{\ell}, \quad B_{j, \sigma}:=\sum_{\ell=0}^{q} B_{\ell, j, \sigma} T^{\ell}, \quad T^{\ell} U_{k}^{m}:=U_{k+\ell}^{m} .
$$

All matrices $A_{\ell}, B_{\ell, j, \sigma}$ in (4) belong to $\mathcal{M}_{D}(\mathbb{R})$ and depend on $\lambda, A, B$ but not on $\Delta t$ (or equivalently $\Delta x$ ). We recall the following definition from [4]:

Definition 2.1 (Strong stability [4). The finite difference approximation (3) is said to be strongly stable if there exists a constant $C$ such that for all $\gamma>0$ and all $\Delta t \in] 0,1]$, the solution $\left(U_{j}^{n}\right)$ of (3) with $f=0$ satisfies the estimate

$$
\begin{aligned}
& \frac{\gamma}{\gamma \Delta t+1} \sum_{n \geq 0} \Delta t \mathrm{e}^{-2 \gamma n \Delta t}\left\|U^{n}\right\|_{1-r,+\infty}^{2}+\sum_{n \geq 0} \Delta t \mathrm{e}^{-2 \gamma n \Delta t} \sum_{j=1-r}^{0}\left|U_{j}^{n}\right|^{2} \\
\leq & C\left\{\frac{\gamma \Delta t+1}{\gamma} \sum_{n \geq 0} \Delta t \mathrm{e}^{-2 \gamma(n+1) \Delta t}\left\|F^{n}\right\|_{1,+\infty}^{2}+\sum_{n \geq 1} \Delta t \mathrm{e}^{-2 \gamma n \Delta t} \sum_{j=1-r}^{0}\left|g_{j}^{n}\right|^{2}\right\} .
\end{aligned}
$$

The estimate in Definition 2.1 is the discrete counterpart of the energy estimate (21) for the "continuous" problem (11) in the case of zero initial data (and when one does not require to control the $L_{t}^{\infty}\left(L_{x}^{2}\right)$ norm of the solution $u$ ). We recall that strong stability in the sense of Definition 2.1 is usually proved by performing a Laplace transform with respect to the time variable. The energy estimate for the resolvent equation is then obtained by using symmetrizers whose construction relies on the so-called uniform Kreiss-Lopatinskii condition (non-existence of unstable nor weakly unstable normal mode). We refer to 4 for some results in this direction. In this paper, we shall assume that the scheme (3) is strongly stable, and we wish to prove an energy estimate for (3) in the case of non-zero initial data. In view of (2), the most obvious space of initial data for (3) is $\ell^{2}$. Let us now introduce our main assumptions, and then state the stability result.

For $\ell=-r, \ldots, p$, and $z \in \mathbb{C} \backslash\{0\}$, we define the matrices

$$
\mathbb{A}_{\ell}(z):=\delta_{\ell 0} I-\frac{1}{z} A_{\ell},
$$

where $\delta_{\ell_{1} \ell_{2}}$ is the Kronecker symbol. We make the following assumption 3 :

Assumption 2.1. The matrix $\mathbb{A}_{p}(z)$ is invertible for all $z \in \mathbb{C}$ with $|z| \geq 1$.

The second crucial assumption is the following:

\footnotetext{
${ }^{2}$ We do not focus here on the construction of such approximations and refer to 3 for some examples that enter this framework; see also Section 5

${ }^{3}$ Assumption 2.1 is similar to [4, Assumption 5.5].
} 
Assumption 2.2. The operator $Q$ satisfies $\|Q v\|_{-\infty,+\infty} \leq\|v\|_{-\infty,+\infty}$ for all $v \in$ $\ell^{2}$, or equivalently,

$$
\forall \xi \in \mathbb{R}, \quad\left|\sum_{\ell=-r}^{p} A_{\ell} \mathrm{e}^{i \ell \xi}\right| \leq 1 .
$$

Our main result is:

Theorem 2.1. Let Assumptions 2.1] and 2.2 be satisfied, and assume that the scheme (3) is strongly stable in the sense of Definition 2.1. Then there exists a constant $C$ such that for all $\gamma>0$ and all $\Delta t \in] 0,1]$, the solution $U$ to (3) satisfies the estimate

$$
\begin{aligned}
& \sup _{n \geq 0} \mathrm{e}^{-2 \gamma n \Delta t}\left\|U^{n}\right\|_{1-r,+\infty}^{2} \\
& +\frac{\gamma}{\gamma \Delta t+1} \sum_{n \geq 0} \Delta t \mathrm{e}^{-2 \gamma n \Delta t}\left\|U^{n}\right\|_{1-r,+\infty}^{2}+\sum_{n \geq 0} \Delta t \mathrm{e}^{-2 \gamma n \Delta t} \sum_{j=1-r}^{0}\left|U_{j}^{n}\right|^{2} \\
& \quad \leq C\left\{\|f\|_{1-r,+\infty}^{2}+\frac{\gamma \Delta t+1}{\gamma} \sum_{n \geq 0} \Delta t \mathrm{e}^{-2 \gamma(n+1) \Delta t}\left\|F^{n}\right\|_{1,+\infty}^{2}\right. \\
& \left.\quad+\sum_{n \geq 1} \Delta t \mathrm{e}^{-2 \gamma n \Delta t} \sum_{j=1-r}^{0}\left|g_{j}^{n}\right|^{2}\right\} .
\end{aligned}
$$

The method of proof is inspired from [17 with, however, some important modifications. More precisely, we introduce an auxiliary discretized problem by modifying the boundary operators $B_{j,-1}, B_{j, 0}$. This auxiliary problem is chosen in such a way that even for non-zero initial data, the solution can be estimated by applying the energy method (to rephrase the terminology of continuous problems, the auxiliary boundary conditions should be "strictly dissipative"). Our auxiliary problem is not the same as in 17]. As a matter of fact, we directly show by means of the energy method that the Dirichlet boundary conditions are strictly dissipative. As announced in the introduction, this is an improved version of the Goldberg-Tadmor Lemma. Our new proof of the Goldberg-Tadmor Lemma can be extended to multidimensional problems even if the equation is not scalar $(D \geq 2)$; see Section 3 (see also Section 4 for variable coefficients). Once we have the estimate for the auxiliary boundary conditions, the end of the proof follows the arguments in [1, Chapter 4] for the continuous problem.

2.2. A refined version of the Goldberg-Tadmor Lemma. In this paragraph, we consider an auxiliary discretization where the (non-homogeneous) Dirichlet conditions are enforced at the boundary:

$$
\begin{cases}V_{j}^{n+1}=Q V_{j}^{n}+\Delta t F_{j}^{n}, & j \geq 1, \quad n \geq 0, \\ V_{j}^{n+1}=g_{j}^{n+1}, & j=1-r, \ldots, 0, \quad n \geq 0, \\ V_{j}^{0}=f_{j}, & j \geq 1-r .\end{cases}
$$

The aim of this paragraph is to prove the following:

Theorem 2.2. Let Assumptions 2.1 and 2.2 be satisfied. Then there exists a constant $C$ such that for all $\gamma>0$ and all $\Delta t \in] 0,1]$, the solution $V$ to (7) satisfies 
the estimate

$$
\begin{aligned}
& \sup _{n \geq 0} \mathrm{e}^{-2 \gamma n \Delta t}\left\|V^{n}\right\|_{1-r,+\infty}^{2}+\frac{\gamma}{\gamma \Delta t+1} \sum_{n \geq 0} \Delta t \mathrm{e}^{-2 \gamma n \Delta t}\left\|V^{n}\right\|_{1-r,+\infty}^{2} \\
& \quad+\sum_{n \geq 0} \Delta t \mathrm{e}^{-2 \gamma n \Delta t} \sum_{j=1-r}^{\max (p, q+1)}\left|V_{j}^{n}\right|^{2} \leq C\left\{\|f\|_{1-r,+\infty}^{2}\right. \\
& \left.+\frac{\gamma \Delta t+1}{\gamma} \sum_{n \geq 0} \Delta t \mathrm{e}^{-2 \gamma(n+1) \Delta t}\left\|F^{n}\right\|_{1,+\infty}^{2}+\sum_{n \geq 1} \Delta t \mathrm{e}^{-2 \gamma n \Delta t} \sum_{j=1-r}^{0}\left|g_{j}^{n}\right|^{2}\right\} .
\end{aligned}
$$

In particular, the discretization (7) is strongly stable in the sense of Definition 2.1.

Theorem 2.2 is an improved version of [2, Lemma 2.3] because we can directly consider non-zero initial data. Moreover, we control in (8) the $\ell_{n}^{\infty}\left(\ell_{j}^{2}\right)$ norm of the solution $V$. This will be useful in the proof of Theorem 2.1.

The key point in the proof of Theorem 2.2 is Assumption 2.2, which can be understood as a symmetry assumption on the matrices $A_{\ell}$. Let us emphasize that Assumption 2.2 can be extended to multidimensional systems (as we shall see in Section 3) while the "scalar" assumption in [2] does not extend to general multidimensional systems.

The proof of Theorem 2.2 is split into several steps. We first observe that the solution $V$ to (7) depends linearly on the source terms $(f, g, F)$. It is therefore sufficient to prove separately (8) in the case $F=0$ (no source term in the interior equation) and in the case $(f, g)=0$ (zero initial data, and homogeneous boundary conditions). It turns out that we use slightly different arguments for both cases. This may look surprising at first glance. We refer to Section 5 for some comments. The proof of Theorem 2.2 below begins with the case $F=0$, and then deals with the case $(f, g)=0$.

Note that in (8), we estimate the weighted $\ell^{2}$-norm in time of the trace $\left(V_{j}^{n}\right)_{n \geq 0}$, for all $j$ from $1-r$ to $\max (p, q+1)$. It would have been sufficient for the proof of Theorem 2.1 to have this type of estimate up to $j=q+1$. Yet, in the proof below, we first obtain an estimate up to $j=p$ (see Corollary 2.1). This is the reason why we have stated (8) this way. We start the proof of Theorem 2.2 with some preliminary results.

Lemma 2.1. Let Assumptions 2.1 and 2.2 be satisfied. Then there exists a constant $C$ such that for all $\gamma>0$ and all $\Delta t \in] 0,1]$, the solution $V$ to (7) with $F=0$ satisfies the estimate

$$
\begin{aligned}
\mathrm{e}^{2 \gamma \Delta t} \sup _{n \geq 1} \mathrm{e}^{-2 \gamma n \Delta t}\left\|V^{n}\right\|_{1,+\infty}^{2}+\gamma \sum_{n \geq 1} \Delta t \mathrm{e}^{-2 \gamma n \Delta t}\left\|V^{n}\right\|_{1,+\infty}^{2} \\
+\sum_{n \geq 0} \Delta t \mathrm{e}^{-2 \gamma n \Delta t} \sum_{j=1-r}^{p}\left|V_{j}^{n}\right|^{2} \\
\quad \leq C\left\{\|f\|_{1-r,+\infty}^{2}+\sum_{n \geq 1} \Delta t \mathrm{e}^{-2 \gamma n \Delta t} \sum_{j=1-r}^{0}\left|g_{j}^{n}\right|^{2}\right\} .
\end{aligned}
$$

Proof of Lemma 2.1. We decompose the operator $Q$ as

$$
Q:=I+\widetilde{Q} .
$$


Assumption 2.2 is equivalent to the inequality

$$
\forall w \in \ell^{2}, \quad 2(w, \widetilde{Q} w)_{-\infty,+\infty}+\|\widetilde{Q} w\|_{-\infty,+\infty}^{2} \leq 0 .
$$

We first use the relation $V_{j}^{n+1}=(I+\widetilde{Q}) V_{j}^{n}$ for $j \geq 1$ (recall that $F=0$ ), and derive

$$
\left\|V^{n+1}\right\|_{1,+\infty}^{2}-\left\|V^{n}\right\|_{1,+\infty}^{2}=2\left(V^{n}, \widetilde{Q} V^{n}\right)_{1,+\infty}+\left\|\widetilde{Q} V^{n}\right\|_{1,+\infty}^{2} .
$$

For a fixed integer $n$, we introduce the sequence $\left(W_{j}\right)_{j \in \mathbb{Z}}$ such that $W_{j}=V_{j}^{n}$ for $j \geq 1-r$ and $W_{j}=0$ for $j \leq-r$. Due to the structure of the operator $\widetilde{Q}$ (see (44)), we have $\widetilde{Q} W_{j}=0$ if $j \leq-r-p$, and $\widetilde{Q} W_{j}=\widetilde{Q} V_{j}^{n}$ if $j \geq 1$. Using (10), we thus get

$$
\begin{aligned}
0 \geq & 2(W, \widetilde{Q} W)_{-\infty,+\infty}+\|\widetilde{Q} W\|_{-\infty,+\infty}^{2} \\
= & 2\left(V^{n}, \widetilde{Q} W\right)_{1-r, 0}+2\left(V^{n}, \widetilde{Q} V^{n}\right)_{1,+\infty} \\
& +\|\widetilde{Q} W\|_{1-r-p,-r}^{2}+\|\widetilde{Q} W\|_{1-r, 0}^{2}+\left\|\widetilde{Q} V^{n}\right\|_{1,+\infty}^{2} \\
= & 2\left(V^{n}, \widetilde{Q} V^{n}\right)_{1,+\infty}+\left\|\widetilde{Q} V^{n}\right\|_{1,+\infty}^{2} \\
& +\left\|V^{n}+\widetilde{Q} W\right\|_{1-r, 0}^{2}+\|\widetilde{Q} W\|_{1-r-p,-r}^{2}-\left\|V^{n}\right\|_{1-r, 0}^{2}
\end{aligned}
$$

We insert (12) into (11) and obtain

$$
\left\|V^{n+1}\right\|_{1,+\infty}^{2}-\left\|V^{n}\right\|_{1,+\infty}^{2}+\|\widetilde{Q} W\|_{1-r-p,-r}^{2}+\left\|V^{n}+\widetilde{Q} W\right\|_{1-r, 0}^{2} \leq\left\|V^{n}\right\|_{1-r, 0}^{2} .
$$

At this point, two situations may occur depending on $p$. Let us first consider the case $p \geq 1$. Then, by Assumption 2.1, $A_{p}$ is an invertible matrix.

Lemma 2.2. Let $p \geq 1$ and let $A_{p}$ be invertible. Then there exists a constant $c>0$ that does not depend on $\Delta t$ nor on $V^{n}$ such that the following estimate holds:

$$
\|\widetilde{Q} W\|_{1-r-p,-r}^{2}+\left\|V^{n}+\widetilde{Q} W\right\|_{1-r, 0}^{2} \geq c\left\|V^{n}\right\|_{1-r, p}^{2} .
$$

Let us assume that Lemma 2.2 holds and go back to (13). We have

$$
\left\|V^{n+1}\right\|_{1,+\infty}^{2}-\left\|V^{n}\right\|_{1,+\infty}^{2}+c \Delta x \sum_{j=1-r}^{p}\left|V_{j}^{n}\right|^{2} \leq \Delta x \sum_{j=1-r}^{0}\left|V_{j}^{n}\right|^{2} .
$$

The end of the proof consists of integrating (14) over $\mathbb{N}$; see a similar calculation in the continous case in [1, page 95]. We let $\gamma>0$ and, for the sake of clarity, we introduce the notation

$$
\begin{aligned}
& \mathcal{V}_{n}:=\mathrm{e}^{-2 \gamma n \Delta t}\left\|V^{n}\right\|_{1,+\infty}^{2} \\
& \mathcal{B}_{n}:=\mathrm{e}^{-2 \gamma n \Delta t} \sum_{j=1-r}^{p}\left|V_{j}^{n}\right|^{2}, \\
& \mathcal{G}_{n}:=\mathrm{e}^{-2 \gamma n \Delta t} \sum_{j=1-r}^{0}\left|V_{j}^{n}\right|^{2} .
\end{aligned}
$$

We multiply (14) by $\exp (-2 \gamma n \Delta t)$ to obtain

$$
\mathrm{e}^{2 \gamma \Delta t} \mathcal{V}_{n+1}-\mathcal{V}_{n}+\frac{c}{\lambda} \Delta t \mathcal{B}_{n} \leq \frac{1}{\lambda} \Delta t \mathcal{G}_{n}
$$


Summing this inequality from 0 to $N$ yields

$$
\begin{aligned}
\mathrm{e}^{2 \gamma \Delta t} \mathcal{V}_{N+1}+\frac{\mathrm{e}^{2 \gamma \Delta t}-1}{\Delta t} \sum_{1}^{N} \Delta t \mathcal{V}_{n} & +\frac{c}{\lambda} \sum_{0}^{N} \Delta t \mathcal{B}_{n} \\
& \leq \mathcal{V}_{0}+\frac{1}{\lambda} \sum_{0}^{N} \Delta t \mathcal{G}_{n} \leq \mathcal{V}_{0}+\frac{1}{\lambda} \sum_{n \geq 0} \Delta t \mathcal{G}_{n}
\end{aligned}
$$

Letting $N$ tend to $+\infty$, we have proved

$$
\mathrm{e}^{2 \gamma \Delta t} \sup _{n \geq 1} \mathcal{V}_{n}+\gamma \sum_{n \geq 1} \Delta t \mathcal{V}_{n}+\sum_{n \geq 0} \Delta t \mathcal{B}_{n} \leq C\left(\mathcal{V}_{0}+\Delta x \mathcal{G}_{0}+\sum_{n \geq 1} \Delta t \mathcal{G}_{n}\right)
$$

The right-hand side of (15) is directly estimated by the right-hand side of (9); see the definition above for $\mathcal{G}_{n}$ and use (7). The constant $C$ in (15) is independent of $\gamma$ and $\Delta t$ and we have therefore completed the proof of (9) in the case $p \geq 1$.

It remains to treat the case $p=0$ for which Lemma 2.2 no longer holds. In this case, we go back to (13) and simply ignore the non-negative "boundary terms" on the left-hand side:

$$
\left\|V^{n+1}\right\|_{1,+\infty}^{2}-\left\|V^{n}\right\|_{1,+\infty}^{2} \leq\left\|V^{n}\right\|_{1-r, 0}^{2} .
$$

We then proceed as above (with the same notation) to derive the weighted-in-time estimate

$$
\mathrm{e}^{2 \gamma \Delta t} \sup _{n \geq 1} \mathcal{V}_{n}+\gamma \sum_{n \geq 1} \Delta t \mathcal{V}_{n} \leq C\left(\mathcal{V}_{0}+\Delta x \mathcal{G}_{0}+\sum_{n \geq 1} \Delta t \mathcal{G}_{n}\right)
$$

In the case $p=0$, the term

$$
\sum_{n \geq 0} \Delta t \mathrm{e}^{-2 \gamma n \Delta t} \sum_{j=1-r}^{p}\left|V_{j}^{n}\right|^{2}
$$

in the left-hand side of (9) is directly estimated by the right-hand side of (9), so that the proof of Lemma 2.1 is complete (provided we prove Lemma 2.2).

Proof of Lemma 2.2. Proving Lemma 2.2 is equivalent to proving that the quadratic form (that is independent on $n$ )

$$
\left(V_{1-r}^{n}, \ldots, V_{p}^{n}\right) \longmapsto \sum_{j=1-r-p}^{-r}\left|\widetilde{Q} W_{j}\right|^{2}+\sum_{j=1-r}^{0}\left|V_{j}^{n}+\widetilde{Q} W_{j}\right|^{2}
$$

is positive definite. Recall that $W$ denotes the extension of $V^{n}$ by zero for $j \leq-r$. The quadratic form (16) is clearly non-negative. Let us therefore consider some vector $\left(V_{1-r}^{n}, \ldots, V_{p}^{n}\right)$ that satisfies

(17) $\forall j=1-r-p, \ldots,-r, \quad \widetilde{Q} W_{j}=0, \quad \forall j=1-r, \ldots, 0, \quad V_{j}^{n}+\widetilde{Q} W_{j}=0$.

We first show by induction on $j$ that $V_{j}^{n}=0$ for all $j=1-r, \ldots, p-r$. Let us recall that $p \geq 1$, so we can write $\widetilde{Q}=Q-I$ in the form

$$
\widetilde{Q}=A_{p} T^{p}+\sum_{\ell=-r}^{p-1} \widetilde{A}_{\ell} T^{\ell}
$$


In particular, we have $\widetilde{Q} W_{1-r-p}=A_{p} V_{1-r}^{n}$, and $V_{1-r}^{n}=0$ because $A_{p}$ is invertible. For $j=1-r-p, \ldots,-r, \widetilde{Q} W_{j}$ equals $A_{p} V_{j+p}^{n}$ plus a linear combination of the $V_{\ell}^{n}, \ell<j+p$. Since the first term $V_{1-r}^{n}$ is zero, we can proceed by induction and get $V_{1-r}^{n}=\cdots=V_{p-r}^{n}=0$.

We now use the second set of equalities in (17). In particular, we have $V_{1-r}^{n}+$ $\widetilde{Q} W_{1-r}=\widetilde{Q} W_{1-r}=A_{p} V_{1-r+p}^{n}$. Therefore, $V_{1-r+p}^{n}=0$, and the rest of the proof follows from another induction argument. We have thus shown that (17) implies $\left(V_{1-r}^{n}, \ldots, V_{p}^{n}\right)=0$. Hence the quadratic form (16) is positive definite. The proof of Lemma 2.2 is complete.

We next turn to the case $(f, g)=0$ with arbitrary interior source $F$.

Lemma 2.3. Let Assumptions 2.1 and 2.2 be satisfied. Then there exists a constant $C$ such that for all $\gamma>0$ and all $\Delta t \in] 0,1]$, the solution $\left(V_{j}^{n}\right)$ to (7) with $(f, g)=0$ satisfies the estimate

$$
\begin{aligned}
& \sup _{n \geq 1} \mathrm{e}^{-2 \gamma n \Delta t}\left\|V^{n}\right\|_{1,+\infty}^{2}+\frac{\gamma}{\gamma \Delta t+1} \sum_{n \geq 1} \Delta t \mathrm{e}^{-2 \gamma n \Delta t}\left\|V^{n}\right\|_{1,+\infty}^{2} \\
& +\mathrm{e}^{-2 \gamma \Delta t} \sum_{n \geq 1} \Delta t \mathrm{e}^{-2 \gamma n \Delta t} \sum_{j=1}^{p}\left|V_{j}^{n}\right|^{2} \\
& \leq C \frac{\gamma \Delta t+1}{\gamma} \sum_{n \geq 0} \Delta t \mathrm{e}^{-2 \gamma(n+1) \Delta t}\left\|F^{n}\right\|_{1,+\infty}^{2} .
\end{aligned}
$$

Proof of Lemma 2.3. Following the proof of Lemma 2.1, we decompose the operator $Q$ as $Q=I+\widetilde{Q}$. We then use the relation $V_{j}^{n+1}=Q V_{j}^{n}+\Delta t F_{j}^{n}$ for $j \geq 1$ to derive

$$
\begin{aligned}
& \left\|V^{n+1}\right\|_{1,+\infty}^{2}-\left\|V^{n}\right\|_{1,+\infty}^{2} \\
& =2\left(V^{n}, \widetilde{Q} V^{n}\right)_{1,+\infty}+\left\|\widetilde{Q} V^{n}\right\|_{1,+\infty}^{2}+2 \Delta t\left(Q V^{n}, F^{n}\right)_{1,+\infty}+\Delta t^{2}\left\|F^{n}\right\|_{1,+\infty}^{2} .
\end{aligned}
$$

Let us first assume that $p$ is positive so that Lemma 2.2 holds. Proceeding as in the proof of Lemma 2.1, we obtain the inequality (recall that here we have homogeneous boundary conditions)

$$
\begin{aligned}
\left\|V^{n+1}\right\|_{1,+\infty}^{2}-\left\|V^{n}\right\|_{1,+\infty}^{2}+ & c \Delta x \sum_{j=1}^{p}\left|V_{j}^{n}\right|^{2} \\
& \leq 2 \Delta t\left\|V^{n}\right\|_{1,+\infty}\left\|F^{n}\right\|_{1,+\infty}+\Delta t^{2}\left\|F^{n}\right\|_{1,+\infty}^{2} .
\end{aligned}
$$

For the sake of clarity, we now introduce the notation

$$
\begin{aligned}
& \mathcal{V}_{n}:=\mathrm{e}^{-2 \gamma n \Delta t}\left\|V^{n}\right\|_{1,+\infty}^{2} \\
& \mathcal{B}_{n}:=\mathrm{e}^{-2 \gamma n \Delta t} \sum_{j=1}^{p}\left|V_{j}^{n}\right|^{2}, \\
& \mathcal{F}_{n}:=\mathrm{e}^{-2 \gamma(n+1) \Delta t}\left\|F^{n}\right\|_{1,+\infty}^{2} .
\end{aligned}
$$

We multiply (19) by $\exp (-2 \gamma(n+1) \Delta t)$ and get

$$
\mathcal{V}_{n+1}-\mathrm{e}^{-2 \gamma \Delta t} \mathcal{V}_{n}+\frac{c}{\lambda} \mathrm{e}^{-2 \gamma \Delta t} \Delta t \mathcal{B}_{n} \leq 2 \Delta t \mathrm{e}^{-\gamma \Delta t} \mathcal{F}_{n}^{1 / 2} \mathcal{V}_{n}^{1 / 2}+\Delta t^{2} \mathcal{F}_{n}
$$


Summing this inequality from 0 to $N$ and recalling that the initial data is zero, we obtain

$$
\begin{aligned}
\mathcal{V}_{N+1}+\frac{1-\mathrm{e}^{-2 \gamma \Delta t}}{\Delta t} \sum_{1}^{N} \Delta t \mathcal{V}_{n}+\frac{c}{\lambda} & \mathrm{e}^{-2 \gamma \Delta t} \sum_{0}^{N} \Delta t \mathcal{B}_{n} \\
& \leq \Delta t \sum_{0}^{N} \Delta t \mathcal{F}_{n}+C \sum_{0}^{N} \sqrt{\Delta t \mathcal{F}_{n}} \sqrt{\Delta t \mathcal{V}_{n}} .
\end{aligned}
$$

Using Young's inequality on the last term of the right-hand side, this becomes

$$
\mathcal{V}_{N+1}+\frac{1-\mathrm{e}^{-2 \gamma \Delta t}}{2 \Delta t} \sum_{0}^{N} \Delta t \mathcal{V}_{n}+\frac{c}{\lambda} \mathrm{e}^{-2 \gamma \Delta t} \sum_{0}^{N} \Delta t \mathcal{B}_{n} \leq C \frac{\Delta t}{1-\mathrm{e}^{-2 \gamma \Delta t}} \sum_{0}^{N} \Delta t \mathcal{F}_{n}
$$

Letting $N$ tend to $+\infty$ then proves (the initial data is zero)

$$
\sup _{n \geq 1} \mathcal{V}_{n}+\frac{\gamma}{\gamma \Delta t+1} \sum_{n \geq 1} \Delta t \mathcal{V}_{n}+\mathrm{e}^{-2 \gamma \Delta t} \sum_{n \geq 1} \Delta t \mathcal{B}_{n} \leq C \frac{\gamma \Delta t+1}{\gamma} \sum_{n \geq 0} \Delta t \mathcal{F}_{n} .
$$

The constant $C$ is independent of $\gamma$ and $\Delta t$.

The case $p=0$ is dealt with in the same way. In this case, the term

$$
\sum_{n \geq 1} \Delta t \mathrm{e}^{-2 \gamma n \Delta t} \sum_{j=1}^{p}\left|V_{j}^{n}\right|^{2},
$$

which appears on the left-hand side of (18), vanishes. The proof of Lemma 2.3 is thus complete.

If we compare Lemma 2.1 to Lemma 2.3, we observe that in (18) the estimate for the trace $\left(V_{j}^{n}\right)_{n \geq 0}, j=1, \ldots, p$, involves a factor $\exp (-2 \gamma \Delta t)$ that deteriorates the estimate when $\gamma \Delta t$ is large. In the following, we derive an additional estimate which enables us to get rid of this factor.

Lemma 2.4. Let Assumptions 2.1 and 2.2 be satisfied. Then there exists a constant $C$ such that for all $\gamma>0$ and all $\Delta t \in] 0,1]$, if $\gamma \Delta t \geq 1$, the solution $\left(V_{j}^{n}\right)$ to (7) with $(f, g)=0$ satisfies the estimate

$$
\sum_{n \geq 1} \Delta t \mathrm{e}^{-2 \gamma n \Delta t} \sum_{j=1}^{p}\left|V_{j}^{n}\right|^{2} \leq C \frac{\gamma \Delta t+1}{\gamma} \sum_{n \geq 0} \Delta t \mathrm{e}^{-2 \gamma(n+1) \Delta t}\left\|F^{n}\right\|_{1,+\infty}^{2} .
$$

Proof of Lemma 2.4, Let $j \in\{1, \ldots, p\}$. We use the relation $V_{j}^{n+1}=Q V_{j}^{n}+\Delta t F_{j}^{n}$ to derive

$$
\left|V_{j}^{n+1}\right|^{2} \leq 2\left|Q V_{j}^{n}\right|^{2}+2 \Delta t^{2}\left|F_{j}^{n}\right|^{2} \leq C\left(\frac{1}{\Delta t}\left\|V^{n}\right\|_{1,+\infty}^{2}+\Delta t\left\|F^{n}\right\|_{1,+\infty}^{2}\right) .
$$

We multiply this inequality by $\exp (-2 \gamma(n+1) \Delta t)$ and sum over $n \geq 0$ :

$$
\begin{aligned}
\sum_{n \geq 1} \Delta t \mathrm{e}^{-2 \gamma n \Delta t}\left|V_{j}^{n}\right|^{2} \leq C\left(\frac{\mathrm{e}^{-2 \gamma \Delta t}}{\Delta t} \sum_{n \geq 1} \Delta t \mathrm{e}^{-2 \gamma n \Delta t}\left\|V^{n}\right\|_{1,+\infty}^{2}\right. & \\
& \left.+\Delta t \sum_{n \geq 0} \Delta t \mathrm{e}^{-2 \gamma(n+1) \Delta t}\left\|F^{n}\right\|_{1,+\infty}^{2}\right) .
\end{aligned}
$$


We now use Lemma 2.3 to estimate the first term of the right-hand side, and obtain

$$
\begin{aligned}
& \sum_{n \geq 1} \Delta t \mathrm{e}^{-2 \gamma n \Delta t}\left|V_{j}^{n}\right|^{2} \\
& \quad \leq C\left(\mathrm{e}^{-2 \gamma \Delta t} \frac{\gamma \Delta t+1}{\gamma \Delta t}+1\right) \frac{\gamma \Delta t+1}{\gamma} \sum_{n \geq 0} \Delta t \mathrm{e}^{-2 \gamma(n+1) \Delta t}\left\|F^{n}\right\|_{1,+\infty}^{2} .
\end{aligned}
$$

The result follows.

The combination of Lemma 2.1 Lemma 2.3 and Lemma 2.4 yields

Corollary 2.1. Let Assumptions 2.1 and 2.2 be satisfied. Then there exists a constant $C$ such that for all $\gamma>0$ and all $\Delta t \in] 0,1]$, the solution $V$ to (7) satisfies the estimate

$$
\begin{aligned}
& \sup _{n \geq 0} \mathrm{e}^{-2 \gamma n \Delta t}\left\|V^{n}\right\|_{1-r,+\infty}^{2}+\frac{\gamma}{\gamma \Delta t+1} \sum_{n \geq 0} \Delta t \mathrm{e}^{-2 \gamma n \Delta t}\left\|V^{n}\right\|_{1-r,+\infty}^{2} \\
& +\sum_{n \geq 0} \Delta t \mathrm{e}^{-2 \gamma n \Delta t} \sum_{j=1-r}^{p}\left|V_{j}^{n}\right|^{2} \leq C\left\{\|f\|_{1-r,+\infty}^{2}\right. \\
& \left.+\frac{\gamma \Delta t+1}{\gamma} \sum_{n \geq 0} \Delta t \mathrm{e}^{-2 \gamma(n+1) \Delta t}\left\|F^{n}\right\|_{1,+\infty}^{2}+\sum_{n \geq 1} \Delta t \mathrm{e}^{-2 \gamma n \Delta t} \sum_{j=1-r}^{0}\left|g_{j}^{n}\right|^{2}\right\} .
\end{aligned}
$$

Proof of Corollary 2.1. First, Lemma 2.1] shows that (20) holds when the interior source term $F$ vanishes. Indeed, (9) implies the weaker inequality

$$
\begin{aligned}
& \sup _{n \geq 1} \mathrm{e}^{-2 \gamma n \Delta t}\left\|V^{n}\right\|_{1,+\infty}^{2} \\
& +\frac{\gamma}{\gamma \Delta t+1} \sum_{n \geq 1} \Delta t \mathrm{e}^{-2 \gamma n \Delta t}\left\|V^{n}\right\|_{1,+\infty}^{2}+\sum_{n \geq 0} \Delta t \mathrm{e}^{-2 \gamma n \Delta t} \sum_{j=1-r}^{p}\left|V_{j}^{n}\right|^{2} \\
& \quad \leq C\left\{\|f\|_{1-r,+\infty}^{2}+\sum_{n \geq 1} \Delta t \mathrm{e}^{-2 \gamma n \Delta t} \sum_{j=1-r}^{0}\left|g_{j}^{n}\right|^{2}\right\} .
\end{aligned}
$$

To obtain (20) in the case $F=0$, it remains to argue that one may replace $\sup _{n \geq 1} \mathrm{e}^{-2 \gamma n \Delta t}\left\|V^{n}\right\|_{1,+\infty}^{2}$ in (21) first by $\sup _{n \geq 1} \mathrm{e}^{-2 \gamma n \Delta t}\left\|V^{n}\right\|_{1-r,+\infty}^{2}$, and then by $\sup _{n \geq 0} \mathrm{e}^{-2 \gamma n \Delta t}\left\|V^{n}\right\|_{1-r,+\infty}^{2}$. Indeed, we have

$$
\mathrm{e}^{-2 \gamma n \Delta t}\left\|V^{n}\right\|_{1-r, 0}^{2}=\lambda \sum_{j=1-r}^{0} \Delta t \mathrm{e}^{-2 \gamma n \Delta t}\left|g_{j}^{n}\right|^{2},
$$

which precisely appears on the right-hand side of (21). It then remains to add the term

$$
\begin{aligned}
\frac{\gamma}{\gamma \Delta t+1} \sum_{n \geq 1} \Delta t \mathrm{e}^{-2 \gamma n \Delta t}\left\|V^{n}\right\|_{1-r, 0}^{2} & =\frac{1}{\lambda} \frac{\gamma \Delta t}{\gamma \Delta t+1} \sum_{n \geq 1} \Delta t \mathrm{e}^{-2 \gamma n \Delta t} \sum_{j=1-r}^{0}\left|V_{j}^{n}\right|^{2} \\
& =\frac{1}{\lambda} \frac{\gamma \Delta t}{\gamma \Delta t+1} \sum_{n \geq 1} \Delta t \mathrm{e}^{-2 \gamma n \Delta t} \sum_{j=1-r}^{0}\left|g_{j}^{n}\right|^{2}
\end{aligned}
$$


to the left-hand side of (21). Changing $C$ accordingly proves the claim.

When the initial data and the boundary source term vanish, estimate (20) is obtained by combining Lemma 2.3 (when $\gamma \Delta t \in] 0,1]$ ) and Lemma 2.4 (when $\gamma \Delta t \geq 1)$. We need not recover the boundary terms in the supremum as above since these boundary terms vanish here.

If we compare the result of Corollary 2.1 with [17, Theorem 3.2], we get better information when $p$ is greater than 2 since we get additional trace estimates. Compared to [17, the present approach has the other advantage of requiring only one auxiliary boundary condition. In [17, the author uses the Goldberg-Tadmor Lemma (and thus Dirichlet boundary conditions) after introducing his own auxiliary boundary operator. Here we only consider the original discretization (3) and the auxiliary discretization (7). We also avoid the use of the GKS theory for proving stability estimates for (17).

Corollary 2.1 proves Theorem 2.2 for $p>q$. In the case $q \geq p$, we need some additional trace estimates. These estimates can be obtained by adapting the method described in [17, page 85]. Actually, the present arguments, based on the energy method, are simpler than those in [17. In particular, we avoid once again the use of the delicate GKS characterization of stability in 4 .

End of the proof of Theorem 2.2. From now on, we consider the case $q \geq p$ since for $q<p$, Corollary 2.1 implies Theorem 2.2. Once again, the proof of (9) is slightly different according to the value of $p$. Let us first assume $p \geq 1$. As in [17, we define the sequence $W_{j}^{n}:=V_{j+1}^{n}$ for $n \geq 0$ and $j \geq 1-r$, which solves the system

$$
\begin{cases}W_{j}^{n+1}=Q W_{j}^{n}+\Delta t F_{j+1}^{n}, & j \geq 1, \quad n \geq 0, \\ W_{j}^{n+1}=g_{j+1}^{n+1}, & j=1-r, \ldots,-1, \quad n \geq 0, \\ W_{0}^{n+1}=V_{1}^{n+1}, & n \geq 0, \\ W_{j}^{0}=f_{j+1}, & j \geq 1-r .\end{cases}
$$

Applying Corollary 2.1 to $W$, we obtain

$$
\begin{aligned}
& \sum_{n \geq 0} \Delta t \mathrm{e}^{-2 \gamma n \Delta t}\left|W_{p}^{n}\right|^{2} \leq C\left\{\frac{\gamma \Delta t+1}{\gamma} \sum_{n \geq 0} \Delta t \mathrm{e}^{-2 \gamma(n+1) \Delta t}\left\|F^{n}\right\|_{2,+\infty}^{2}\right. \\
& \left.\quad+\|f\|_{2-r,+\infty}^{2}+\sum_{n \geq 1} \Delta t \mathrm{e}^{-2 \gamma n \Delta t} \sum_{j=2-r}^{0}\left|g_{j}^{n}\right|^{2}+\sum_{n \geq 1} \Delta t \mathrm{e}^{-2 \gamma n \Delta t}\left|V_{1}^{n}\right|^{2}\right\} .
\end{aligned}
$$

Using again Corollary 2.1 to estimate the last term of the right-hand side (this is possible because $p \geq 1$ ) yields

$$
\begin{aligned}
\sum_{n \geq 0} \Delta t \mathrm{e}^{-2 \gamma n \Delta t}\left|V_{p+1}^{n}\right|^{2} \leq C\{ & \frac{\gamma \Delta t+1}{\gamma} \sum_{n \geq 0} \Delta t \mathrm{e}^{-2 \gamma(n+1) \Delta t}\left\|F^{n}\right\|_{1,+\infty}^{2} \\
& \left.+\|f\|_{1-r,+\infty}^{2}+\sum_{n \geq 1} \Delta t \mathrm{e}^{-2 \gamma n \Delta t} \sum_{j=1-r}^{0}\left|g_{j}^{n}\right|^{2}\right\} .
\end{aligned}
$$


We have therefore derived a trace estimate for $\left(V_{p+1}^{n}\right)_{n \geq 0}$. A straightforward induction argument gives

$$
\begin{gathered}
\sum_{n \geq 0} \Delta t \mathrm{e}^{-2 \gamma n \Delta t} \sum_{j=p+1}^{q+1}\left|V_{j}^{n}\right|^{2} \leq C\left\{\frac{\gamma \Delta t+1}{\gamma} \sum_{n \geq 0} \Delta t \mathrm{e}^{-2 \gamma(n+1) \Delta t}\left\|F^{n}\right\|_{1,+\infty}^{2}\right. \\
\left.+\|f\|_{1-r,+\infty}^{2}+\sum_{n \geq 1} \Delta t \mathrm{e}^{-2 \gamma n \Delta t} \sum_{j=1-r}^{0}\left|g_{j}^{n}\right|^{2}\right\} .
\end{gathered}
$$

The combination of (20) and (22) proves the main stability estimate (9) for $p \geq 1$.

We now consider the case $p=0$. Since Corollary 2.1 does not give any trace estimate for $\left(V_{j}^{n}\right)_{n \geq 0}$ with $j \geq 1$, the shift argument of [17 cannot be used as above. From Assumption 2.1, we know that the spectral radius of $A_{0}$ is strictly less than 1. Hence, there exist a positive definite symmetric matrix $H$ and a positive number $\varepsilon_{0}$ such that if we consider the new Euclidean norm on $\mathbb{R}^{D}$,

$$
\forall X \in \mathbb{R}^{D}, \quad|X|_{H}:=\sqrt{X^{*} H X},
$$

then we have

$$
\forall X \in \mathbb{R}^{D}, \quad\left|A_{0} X\right|_{H} \leq \sqrt{1-2 \varepsilon_{0}}|X|_{H} .
$$

From the relation

$$
V_{1}^{n+1}=A_{0} V_{1}^{n}+\sum_{\ell=-r}^{-1} A_{\ell} V_{1+\ell}^{n}+\Delta t F_{1}^{n}=A_{0} V_{1}^{n}+\underbrace{\sum_{j=1-r}^{0} A_{j-1} g_{j}^{n}+\Delta t F_{1}^{n}}_{=: X^{n}},
$$

where we use the notation $g_{j}^{0}:=f_{j}$ for $j=1-r, \ldots, 0$, we get

$$
\begin{aligned}
\left|V_{1}^{n+1}\right|_{H}^{2} & =\left|A_{0} V_{1}^{n}\right|_{H}^{2}+2\left(A_{0} V_{1}^{n}\right)^{*} H X^{n}+\left|X^{n}\right|_{H}^{2} \\
& \leq\left(1-2 \varepsilon_{0}\right)\left|V_{1}^{n}\right|_{H}^{2}+2\left(A_{0} V_{1}^{n}\right)^{*} H X^{n}+\left|X^{n}\right|_{H}^{2} \\
& \leq\left(1-\varepsilon_{0}\right)\left|V_{1}^{n}\right|_{H}^{2}+\left(1+\varepsilon_{0}^{-1}\right)\left|X^{n}\right|_{H}^{2} .
\end{aligned}
$$

By the definition of $X^{n}$, this becomes

$$
\left|V_{1}^{n+1}\right|_{H}^{2}-\left|V_{1}^{n}\right|_{H}^{2}+\varepsilon_{0}\left|V_{1}^{n}\right|_{H}^{2} \leq C\left(\Delta t\left\|F^{n}\right\|_{1,+\infty}^{2}+\sum_{j=1-r}^{0}\left|g_{j}^{n}\right|^{2}\right) .
$$

Using the same summation process as earlier, we obtain

$$
\begin{gathered}
\left\{\left(1-\mathrm{e}^{-2 \gamma \Delta t}\right)+\varepsilon_{0} \mathrm{e}^{-2 \gamma \Delta t}\right\} \sum_{n \geq 0} \Delta t \mathrm{e}^{-2 \gamma n \Delta t}\left|V_{1}^{n}\right|_{H}^{2} \\
\leq C\left\{\|f\|_{1-r,+\infty}^{2}+\frac{\gamma \Delta t+1}{\gamma} \sum_{n \geq 0} \Delta t \mathrm{e}^{-2 \gamma(n+1) \Delta t}\left\|F^{n}\right\|_{1,+\infty}^{2}\right. \\
\left.\quad+\sum_{n \geq 1} \Delta t \mathrm{e}^{-2 \gamma n \Delta t} \sum_{j=1-r}^{0}\left|g_{j}^{n}\right|^{2}\right\} .
\end{gathered}
$$


The norm $|\cdot|_{H}$ and the standard Euclidean norm are equivalent, so that

$$
\begin{aligned}
\sum_{n \geq 0} \Delta t \mathrm{e}^{-2 \gamma n \Delta t}\left|V_{1}^{n}\right|^{2} \leq C & \left\{\frac{\gamma \Delta t+1}{\gamma} \sum_{n \geq 0} \Delta t \mathrm{e}^{-2 \gamma(n+1) \Delta t}\left\|F^{n}\right\|_{1,+\infty}^{2}\right. \\
& \left.+\|f\|_{1-r,+\infty}^{2}+\sum_{n \geq 1} \Delta t \mathrm{e}^{-2 \gamma n \Delta t} \sum_{j=1-r}^{0}\left|g_{j}^{n}\right|^{2}\right\},
\end{aligned}
$$

with a constant $C$ that does not depend on $\gamma$ nor on $\Delta t$. The proof of (9) follows from an induction argument where we apply the above method to recover the estimate for the trace $\left(V_{j}^{n}\right)_{n \geq 0}, j=2, \ldots, q+1$. The proof of Theorem 2.2 is now complete.

2.3. Proof of Theorem 2.1. We rewrite the solution $U$ to (3) as $U=V+W$, where $V$ satisfies

$$
\begin{cases}V_{j}^{n+1}=Q V_{j}^{n}+\Delta t F_{j}^{n}, & j \geq 1, \quad n \geq 0, \\ V_{j}^{n+1}=g_{j}^{n+1}, & j=1-r, \ldots, 0, \quad n \geq 0, \\ V_{j}^{0}=f_{j}, & j \geq 1-r,\end{cases}
$$

and $W$ satisfies

$$
\begin{cases}W_{j}^{n+1}=Q W_{j}^{n}, & j \geq 1, \quad n \geq 0, \\ W_{j}^{n+1}=B_{j,-1} W_{1}^{n+1}+B_{j, 0} W_{1}^{n}+\widetilde{g}_{j}^{n+1}, & j=1-r, \ldots, 0, \quad n \geq 0, \\ W_{j}^{0}=0, & j \geq 1-r .\end{cases}
$$

The source term $\widetilde{g}$ in (24) is defined by

$$
\forall j=1-r, \ldots, 0, \quad \forall n \geq 1, \quad \widetilde{g}_{j}^{n}:=B_{j,-1} V_{1}^{n}+B_{j, 0} V_{1}^{n-1} .
$$

The estimate for $V$ is given by Theorem 2.2. In addition, since the discretization (3) is strongly stable in the sense of Definition 2.1 and the initial data in (24) is zero, $W$ satisfies

$$
\begin{array}{r}
\frac{\gamma}{\gamma \Delta t+1} \sum_{n \geq 0} \Delta t \mathrm{e}^{-2 \gamma n \Delta t}\left\|W^{n}\right\|_{1-r,+\infty}^{2}+\sum_{n \geq 0} \Delta t \mathrm{e}^{-2 \gamma n \Delta t} \sum_{j=1-r}^{0}\left|W_{j}^{n}\right|^{2} \\
\leq C \sum_{n \geq 1} \Delta t \mathrm{e}^{-2 \gamma n \Delta t} \sum_{j=1-r}^{0}\left|\widetilde{g}_{j}^{n}\right|^{2} .
\end{array}
$$

The defining equation (25) together with (8) allow us to control the term involving $\widetilde{g}_{j}^{n}$ by

$$
\begin{aligned}
\sum_{n \geq 1} \Delta t \mathrm{e}^{-2 \gamma n \Delta t} \sum_{j=1-r}^{0}\left|\widetilde{g}_{j}^{n}\right|^{2} & \leq C\left\{\frac{\gamma \Delta t+1}{\gamma} \sum_{n \geq 0} \Delta t \mathrm{e}^{-2 \gamma(n+1) \Delta t}\left\|F^{n}\right\|_{1,+\infty}^{2}\right. \\
& \left.+\|f\|_{1-r,+\infty}^{2}+\sum_{n \geq 1} \Delta t \mathrm{e}^{-2 \gamma n \Delta t} \sum_{j=1-r}^{0}\left|g_{j}^{n}\right|^{2}\right\}
\end{aligned}
$$


Hence, we obtain

$$
\begin{aligned}
& \frac{\gamma}{\gamma \Delta t+1} \sum_{n \geq 0} \Delta t \mathrm{e}^{-2 \gamma n \Delta t}\left\|W^{n}\right\|_{1-r,+\infty}^{2}+ \sum_{n \geq 0} \Delta t \mathrm{e}^{-2 \gamma n \Delta t} \sum_{j=1-r}^{0}\left|W_{j}^{n}\right|^{2} \\
& \leq C\left\{\|f\|_{1-r,+\infty}^{2}+\frac{\gamma \Delta t+1}{\gamma} \sum_{n \geq 0} \Delta t \mathrm{e}^{-2 \gamma(n+1) \Delta t}\left\|F^{n}\right\|_{1,+\infty}^{2}\right. \\
&\left.+\sum_{n \geq 1} \Delta t \mathrm{e}^{-2 \gamma n \Delta t} \sum_{j=1-r}^{0}\left|g_{j}^{n}\right|^{2}\right\} .
\end{aligned}
$$

The combination of estimate (27) for $W$ and estimate (8) for $V$ proves the first part of Theorem 2.1. To complete the proofs, it remains to control the $\ell_{n}^{\infty}\left(\ell_{j}^{2}\right)$ norm of $W$. This is done by following the proof of Lemma 2.1. More precisely, we have

Lemma 2.5. Let Assumption 2.2 be satisfied, and assume that the discretization (31) is strongly stable in the sense of Definition 2.1. Then there exists a constant $C$ that does not depend on the data $\widetilde{g}$ such that for all $\gamma>0$ and all $\Delta t \in] 0,1]$, the solution $W$ to (24) satisfies

$$
\sup _{n \geq 0} \mathrm{e}^{-2 \gamma n \Delta t}\left\|W^{n}\right\|_{1-r,+\infty}^{2} \leq C \sum_{n \geq 1} \Delta t \mathrm{e}^{-2 \gamma n \Delta t} \sum_{j=1-r}^{0}\left|\widetilde{g}_{j}^{n}\right|^{2} .
$$

Writing $U=V+W$, the estimates of Theorem 2.2 and Lemma 2.5 combined with (26), (27) yield (6). Therefore, it only remains to prove Lemma 2.5. The proof of the corresponding result in [17] relies on the GKS theory and uses a Laplace transform in time. This hardly applies for variable coefficients as considered in Section 4 We therefore propose an elementary proof of Lemma 2.5 based on the energy method.

Proof of Lemma 2.5. We start from (24) and apply the strategy of Lemma 2.1. Since the derivation of the inequality (13) only relies on Assumption 2.2 and not on the boundary operator, we have (just ignore the non-negative boundary terms on the left-hand side of (13))

$$
\left\|W^{n+1}\right\|_{1,+\infty}^{2}-\left\|W^{n}\right\|_{1,+\infty}^{2} \leq\left\|W^{n}\right\|_{1-r, 0}^{2} .
$$

We multiply this inequality by $\exp (-2 \gamma n \Delta t)$ and use the summation process as in the proof of Lemma 2.1. Since the initial data for (24) vanish, this yields

$$
\sup _{n \geq 0} \mathrm{e}^{-2 \gamma n \Delta t}\left\|W^{n}\right\|_{1,+\infty}^{2} \leq C \sum_{n \geq 1} \Delta t \mathrm{e}^{-2 \gamma n \Delta t} \sum_{j=1-r}^{0}\left|W_{j}^{n}\right|^{2} .
$$

We now use the strong stability of the scheme (3) in the sense of Definition 2.1] This allows us to control the right-hand side of (28), and we get

$$
\sup _{n \geq 0} \mathrm{e}^{-2 \gamma n \Delta t}\left\|W^{n}\right\|_{1,+\infty}^{2} \leq C \sum_{n \geq 1} \Delta t \mathrm{e}^{-2 \gamma n \Delta t} \sum_{j=1-r}^{0}\left|\widetilde{g}_{j}^{n}\right|^{2} .
$$


In order to complete the proof of Lemma 2.5, it remains to observe that

$$
\begin{aligned}
& \sup _{n \geq 0} \mathrm{e}^{-2 \gamma n \Delta t}\left\|W^{n}\right\|_{1-r, 0}^{2}=\sup _{n \geq 1} \Delta x \mathrm{e}^{-2 \gamma n \Delta t} \sum_{j=1-r}^{0}\left|W_{j}^{n}\right|^{2} \\
& \leq C \sum_{n \geq 1} \Delta t \mathrm{e}^{-2 \gamma n \Delta t} \sum_{j=1-r}^{0}\left|W_{j}^{n}\right|^{2} \leq C \sum_{n \geq 1} \Delta t \mathrm{e}^{-2 \gamma n \Delta t} \sum_{j=1-r}^{0}\left|\widetilde{g}_{j}^{n}\right|^{2},
\end{aligned}
$$

where we use once again the strong stability of (3) and the fact that the initial data for (24) vanish.

\section{Multidimensional problems: Constant coefficients}

For multidimensional problems, we need further notation for norms on $\ell^{2}\left(\mathbb{Z}^{d}\right)$. Let $\Delta x_{i}>0$ for $i=1, \ldots, d$ be $d$ space steps. For all integers $m_{1} \leq m_{2}$, we set

$$
\|u\|_{m_{1}, m_{2}}^{2}:=\Delta x_{1} \sum_{j_{1}=m_{1}}^{m_{2}}\left(\prod_{k=2}^{d} \Delta x_{k}\right) \sum_{i=2}^{d} \sum_{j_{i} \in \mathbb{Z}}\left|u_{j_{1}, \ldots, j_{d}}\right|^{2},
$$

to denote the $\ell^{2}$-norm on the set $\left[m_{1}, m_{2}\right] \times \mathbb{Z}^{d-1}\left(m_{1}\right.$ may equal $-\infty$ and $m_{2}$ may equal $+\infty)$.

We shall also make use of the $\ell^{2}\left(\mathbb{Z}^{d-1}\right)$-norm that we denote by $\|\cdot\|$ : for all $v \in \ell^{2}\left(\mathbb{Z}^{d-1}\right)$,

$$
\|v\|^{2}:=\left(\prod_{k=2}^{d} \Delta x_{k}\right) \sum_{i=2}^{d} \sum_{j_{i} \in \mathbb{Z}}\left|v_{j_{2}, \ldots, j_{d}}\right|^{2} .
$$

3.1. Main result in several space dimensions. We consider the hyperbolic initial-boundary value problem corresponding to (1) in several space dimensions $d>1$; that is,

$$
\begin{cases}\partial_{t} u+\sum_{i=1}^{d} A_{i} \partial_{x_{i}} u=F(t, x), & (t, x) \in \mathbb{R}^{+} \times \mathbb{R}_{+}^{d}, \\ B u\left(t,\left(0, x^{\prime}\right)\right)=g\left(t, x^{\prime}\right), & t \in \mathbb{R}^{+}, x^{\prime} \in \mathbb{R}^{d-1}, \\ u(0, x)=f(x), & x \in \mathbb{R}_{+}^{d},\end{cases}
$$

where $\mathbb{R}_{+}^{d}:=\mathbb{R}^{+} \times \mathbb{R}^{d-1}$, the matrices $A_{i} \in \mathcal{M}_{D}(\mathbb{R})$ are such that the symbol $\mathbb{R}^{d} \ni \xi \mapsto A(\xi):=\sum_{i=1}^{d} \xi_{i} A_{i}$ is uniformly diagonalizable (see [1, Theorem 1.3]) in $\mathbb{R}$, and $B \in \mathcal{M}_{D_{+}, D}(\mathbb{R})$ with $D_{+}$the number of positive eigenvalues of $A_{1}$. We assume that the boundary is non-characteristic, that is, $0 \notin \operatorname{sp}\left(A_{1}\right)$. Problem (29) is strongly well posed if and only if the matrices $\left\{A_{i}\right\}$ and $B$ satisfy the socalled uniform Kreiss-Lopatinskii condition. In that case, the solution $u$ to (29) belongs to $\mathcal{C}\left(\mathbb{R}^{+} ; L^{2}\left(\mathbb{R}_{+}^{d}\right)\right)$ and its trace on $\left\{x_{1}=0\right\}$ is well defined and belongs to 
$\mathrm{e}^{\gamma t} L^{2}\left(\mathbb{R}^{+} ; L^{2}\left(\mathbb{R}^{d-1}\right)\right)$ for all $\gamma>0$. Moreover, for all $\gamma>0$, $u$ satisfies the energy estimate

$$
\begin{aligned}
& \sup _{t \geq 0} \mathrm{e}^{-2 \gamma t}\|u(t, \cdot)\|_{L^{2}\left(\mathbb{R}_{+}^{d}\right)}^{2}+\gamma \int_{0}^{+\infty} \mathrm{e}^{-2 \gamma t}\|u(t, \cdot)\|_{L^{2}\left(\mathbb{R}_{+}^{d}\right)}^{2} \mathrm{~d} t \\
& \quad+\int_{0}^{+\infty} \mathrm{e}^{-2 \gamma t}\|u(t,(0, \cdot))\|_{L^{2}\left(\mathbb{R}^{d-1}\right)}^{2} \mathrm{~d} t \leq C\left(\|f\|_{L^{2}\left(\mathbb{R}_{+}^{d}\right)}^{2}\right. \\
& \left.\quad+\frac{1}{\gamma} \int_{0}^{+\infty} \mathrm{e}^{-2 \gamma t}\|F(t, \cdot)\|_{L^{2}\left(\mathbb{R}_{+}^{d}\right)}^{2} \mathrm{~d} t+\int_{0}^{+\infty} \mathrm{e}^{-2 \gamma t}\|g(t, \cdot)\|_{L^{2}\left(\mathbb{R}^{d-1}\right)}^{2} \mathrm{~d} t\right),
\end{aligned}
$$

where the constant $C$ is independent of $\gamma, f, F, g$.

As for the one-dimensional case, we introduce the finite difference approximation of (29). We denote by $\Delta x:=\left\{\Delta x_{i}\right\}_{i=1, \ldots, d}$ and $\Delta t$ the space and time steps related by the fixed ratios $\lambda_{i}:=\Delta t / \Delta x_{i}$. For all $j \in \mathbb{Z}^{d}$, we set $j=\left(j_{1}, j^{\prime}\right)$ with $j^{\prime}=\left(j_{2}, \ldots, j_{d}\right)$. We let $p, q, r \in \mathbb{N}^{d}$ be some multi-integers, and define $p_{1}, q_{1}, r_{1}$, $p^{\prime}, q^{\prime}, r^{\prime}$ according to the above notation. The solution $u$ to (29) is approximated by a sequence $\left(U_{j}^{n}\right)=\left(U_{j_{1}, j^{\prime}}^{n}\right)$ for $n \in \mathbb{N}, j_{1} \in 1-r_{1}+\mathbb{N}$, and $j^{\prime} \in \mathbb{Z}^{d-1}$. For $j_{1}=$ $1-r_{1}, \ldots, 0, U_{j_{1}}^{n}$. approximates the trace $u(n \Delta t, 0, \cdot)$ on the boundary $\left\{x_{1}=0\right\}$, and possibly the trace of normal derivatives. We consider one-step finite difference approximations of (29) of the form

$$
\left\{\begin{aligned}
U_{j_{1}, j^{\prime}}^{n+1}= & Q U_{j_{1}, j^{\prime}}^{n}+\Delta t F_{j_{1}, j^{\prime}}^{n}, & & j_{1} \geq 1, j^{\prime} \in \mathbb{Z}^{d-1}, \quad n \geq 0, \\
U_{j_{1}, j^{\prime}}^{n+1}= & B_{j_{1},-1} U_{1, j^{\prime}}^{n+1} & & \\
& +B_{j_{1}, 0} U_{1, j^{\prime}}^{n}+g_{j_{1}, j^{\prime}}^{n+1}, & & 1-r_{1} \leq j_{1} \leq 0, j^{\prime} \in \mathbb{Z}^{d-1}, n \geq 0, \\
U_{j_{1}, j^{\prime}}^{0}= & f_{j_{1}, j^{\prime}}, & & j_{1} \geq 1-r_{1}, j^{\prime} \in \mathbb{Z}^{d-1},
\end{aligned}\right.
$$

where the operators $Q, B_{j_{1},-1}, B_{j_{1}, 0}$ are now given by

$$
\begin{aligned}
& Q:=\sum_{\ell_{1}=-r_{1}}^{p_{1}}\left(\sum_{\ell^{\prime}=-r^{\prime}}^{p^{\prime}} A_{\ell_{1}, \ell^{\prime}} T^{\prime \ell^{\prime}}\right) T^{\ell_{1}}, \\
& B_{j_{1}, \sigma}:=\sum_{\ell_{1}=0}^{q_{1}}\left(\sum_{\ell^{\prime}=-q^{\prime}}^{q^{\prime}} B_{\ell_{1}, \ell^{\prime}, j_{1}, \sigma} T^{\ell^{\prime}}\right) T^{\ell_{1}}, \\
& T^{\ell_{1}} U_{k_{1}, k^{\prime}}^{m}:=U_{k_{1}+\ell_{1}, k^{\prime}}^{m}, \\
& T^{\ell^{\prime}} U_{k_{1}, k^{\prime}}^{m}:=U_{k_{1}, k^{\prime}+\ell^{\prime}}^{m} .
\end{aligned}
$$

In (32), the matrices $A_{\ell}, B_{\ell, j_{1}, \sigma}$ belong to $\mathcal{M}_{D}(\mathbb{R})$ and depend on $\left\{\lambda_{i}, A_{i}\right\}_{i=1, \ldots, d}, B$ but not on $\Delta t$ (or equivalently not on $\Delta x$ ). For multidimensional problems, the notion of strong stability now reads:

Definition 3.1 (Strong stability [11). The finite difference approximation (31) is said to be strongly stable if there exists a constant $C$ such that for all $\gamma>0$ and 
all $\Delta t \in] 0,1]$, the solution $\left(U_{j}^{n}\right)$ of (3) with $f=0$ satisfies the estimate

$$
\begin{aligned}
& \frac{\gamma}{\gamma \Delta t+1} \sum_{n \geq 0} \Delta t \mathrm{e}^{-2 \gamma n \Delta t}\left\|U^{n}\right\|_{1-r_{1},+\infty}^{2}+\sum_{n \geq 0} \Delta t \mathrm{e}^{-2 \gamma n \Delta t} \sum_{j_{1}=1-r_{1}}^{0}\left\|U_{j_{1},}^{n}\right\|^{2} \\
& \leq C\left\{\frac{\gamma \Delta t+1}{\gamma} \sum_{n \geq 0} \Delta t \mathrm{e}^{-2 \gamma(n+1) \Delta t}\left\|F^{n}\right\|_{1,+\infty}^{2}\right. \\
&\left.+\sum_{n \geq 1} \Delta t \mathrm{e}^{-2 \gamma n \Delta t} \sum_{j_{1}=1-r_{1}}^{0}\left\|g_{j_{1},}^{n}\right\|^{2}\right\} .
\end{aligned}
$$

We are now in a position to introduce the hypotheses corresponding to Assumptions 2.1 and 2.2 in the multidimensional case. For $\ell_{1}=-r_{1}, \ldots, p_{1}$, and $z \in \mathbb{C} \backslash\{0\}$, let us define the linear mappings

$$
\begin{aligned}
\mathbb{A}_{\ell_{1}}(z): \quad \ell^{2}\left(\mathbb{Z}^{d-1}\right) & \rightarrow \ell^{2}\left(\mathbb{Z}^{d-1}\right) \\
w & \mapsto \delta_{\ell_{1} 0} w-\frac{1}{z} \sum_{\ell^{\prime}=-r^{\prime}}^{p^{\prime}} A_{\ell_{1}, \ell^{\prime}} T^{\prime \ell^{\prime}} w .
\end{aligned}
$$

We make the following first assumption:

Assumption 3.1. The mapping $\mathbb{A}_{p_{1}}(z)$ is coercive on $\ell^{2}\left(\mathbb{Z}^{d-1}\right)$ for all $z \in \mathbb{C}$ with $|z| \geq 1$. More precisely, there exists a constant $c>0$ such that for all $z \in \mathbb{C}$ with $|z| \geq 1$ and for all $w \in \ell^{2}\left(\mathbb{Z}^{d-1}\right)$, we have

$$
\left\|\mathbb{A}_{p_{1}}(z) w\right\| \geq \frac{c}{|z|^{\nu}}\|w\|, \quad \nu:= \begin{cases}1 & \text { if } p_{1}>0 \\ 0 & \text { otherwise }\end{cases}
$$

Remark 3.1. If $p_{1}=0$, then Assumption 3.1 amounts to assuming that $\mathbb{A}_{0}(z)$ is an isomorphism on $\ell^{2}\left(\mathbb{Z}^{d-1}\right)$ for all $z \in \mathbb{C}$ with $|z| \geq 1$.

For $p_{1}>0$, Assumption 3.1 is slightly weaker than assuming that $\mathbb{A}_{p_{1}}(z)$ is an isomorphism since the fulfillment of Assumption 3.1 does not necessarily imply the surjectivity of $\mathbb{A}_{p_{1}}(z)$ on $\ell^{2}\left(\mathbb{Z}^{d-1}\right)$.

The second assumption is unchanged:

Assumption 3.2. The operator $Q$ satisfies $\|Q v\|_{-\infty,+\infty} \leq\|v\|_{-\infty,+\infty}$ for all $v \in$ $\ell^{2}\left(\mathbb{Z}^{d}\right)$, or equivalently

$$
\forall \xi=\left(\xi_{1}, \xi^{\prime}\right) \in \mathbb{R} \times \mathbb{R}^{d-1}, \quad\left|\sum_{\ell_{1}=-r_{1}}^{p_{1}}\left(\sum_{\ell^{\prime}=-r^{\prime}}^{p^{\prime}} A_{\ell_{1}, \ell^{\prime}} e^{i \ell^{\prime} \cdot \xi^{\prime}}\right) e^{i \ell_{1} \xi_{1}}\right| \leq 1 .
$$

We are now in a position to state the stability result corresponding to Theorem 2.1 in the multidimensional case.

Theorem 3.1. Let Assumptions 3.1 and 3.2 be satisfied, and assume that the scheme (31) is strongly stable in the sense of Definition 3.1. Then there exists a constant $C$ such that for all $\gamma>0$ and all $\Delta t \in] 0,1]$, the solution $U$ to (31) satisfies 
the estimate

$$
\begin{gathered}
\sup _{n \geq 0} \mathrm{e}^{-2 \gamma n \Delta t}\left\|U^{n}\right\|_{1-r_{1},+\infty}^{2}+\frac{\gamma}{\gamma \Delta t+1} \sum_{n \geq 0} \Delta t \mathrm{e}^{-2 \gamma n \Delta t}\left\|U^{n}\right\|_{1-r_{1},+\infty}^{2} \\
+\sum_{n \geq 0} \Delta t \mathrm{e}^{-2 \gamma n \Delta t} \sum_{j_{1}=1-r_{1}}^{0}\left\|U_{j_{1},}^{n}\right\|^{2} \leq C\left\{\|f\|_{1-r_{1},+\infty}^{2}\right. \\
\left.+\frac{\gamma \Delta t+1}{\gamma} \sum_{n \geq 0} \Delta t \mathrm{e}^{-2 \gamma(n+1) \Delta t}\left\|F^{n}\right\|_{1,+\infty}^{2}+\sum_{n \geq 1} \Delta t \mathrm{e}^{-2 \gamma n \Delta t} \sum_{j_{1}=1-r_{1}}^{0}\left\|g_{j_{1},}^{n}\right\|^{2}\right\} .
\end{gathered}
$$

To prove Theorem [3.1, one could mimic the proof of Theorem 2.1 and write the analogues of Lemmas 2.1] 2.2. etc. The proofs would work exactly the same way. The only subtlety would be the extension of Lemma 2.2. For one-dimensional problems, Lemma 2.2 is a result for quadratic forms on a finite dimensional space. The analogue for multidimensional problems would be a result for quadratic forms on an infinite dimensional space. In infinite dimension, however, a quadratic form is not necessarily positive definite if its isotropic cone is trivial. Hence one should pay a little attention. We refer to Section 4 for such a strategy of proof of the results of Section 2 in several space dimensions with variable coefficients. In the present section, we shall show that Theorem 3.1 can be easily obtained by combining Theorem 2.1 and a partial Fourier transform. This approach is specific to the constant coefficients case. It has the advantage to clarify why Assumptions 3.1 and 3.2 are the natural counterparts of Assumptions 2.1 and 2.2 in several space dimensions.

Proof of Theorem 3.1. Let us start with the scheme (31) defining the sequence $\left(U_{j}^{n}\right)$. For each pair $\left(j_{1}, n\right)$ with $j_{1} \geq 1-r_{1}$ and $n \geq 0$, we define the piecewise constant function $V_{j_{1}}^{n}\left(x^{\prime}\right)$ on $\mathbb{R}^{d-1}$ in such a way that $V_{j_{1}}^{n}\left(x^{\prime}\right)$ equals $U_{j_{1}, j^{\prime}}^{n}$ on the mesh element with index $j^{\prime} \in \mathbb{Z}^{d-1}$. We then define the functions $F_{j_{1}}^{n}, g_{j_{1}}^{n}, f_{j_{1}}$ in a similar way. Applying a partial Fourier transform in the tangential variables $x^{\prime}$, equation (31) becomes

$$
\left\{\begin{array}{rlrl}
\widehat{V_{j_{1}}^{n+1}}\left(\xi^{\prime}\right)=Q\left(\theta^{\prime}\right) \widehat{V_{j_{1}}^{n}}\left(\xi^{\prime}\right)+\Delta t \widehat{F_{j_{1}}^{n}}\left(\xi^{\prime}\right), & & j_{1} \geq 1, \quad n \geq 0, \\
\widehat{V_{j_{1}}^{n+1}}\left(\xi^{\prime}\right)=B_{j_{1},-1}\left(\theta^{\prime}\right) \widehat{V_{1}^{n+1}}\left(\xi^{\prime}\right) & \widehat{ } & \\
& +B_{j_{1}, 0}\left(\theta^{\prime}\right) \widehat{V_{1}^{n}}\left(\xi^{\prime}\right)+\widehat{g_{j_{1}}^{n+1}}\left(\xi^{\prime}\right), & & 1-r_{1} \leq j_{1} \leq 0, \quad n \geq 0, \\
\widehat{V_{j_{1}}}\left(\xi^{\prime}\right)=\widehat{f_{j_{1}}}\left(\xi^{\prime}\right), & & j_{1} \geq 1-r_{1},
\end{array}\right.
$$

where $\xi^{\prime}=\left(\xi_{2}, \ldots, \xi_{d}\right) \in \mathbb{R}^{d-1}$ denotes the frequency variables, $\theta^{\prime} \in \mathbb{R}^{d-1}$ is a short notation for the vector $\left(\xi_{2} \Delta x_{2}, \ldots, \xi_{d} \Delta x_{d}\right)$, and the operators $Q\left(\theta^{\prime}\right), B_{j_{1},-1}\left(\theta^{\prime}\right)$, $B_{j_{1}, 0}\left(\theta^{\prime}\right)$ are defined by

$$
\begin{aligned}
& Q\left(\theta^{\prime}\right):=\sum_{\ell_{1}=-r_{1}}^{p_{1}}\left(\sum_{\ell^{\prime}=-r^{\prime}}^{p^{\prime}} A_{\ell_{1}, \ell^{\prime}} \mathrm{e}^{i \ell^{\prime} \cdot \theta^{\prime}}\right) T^{\ell_{1}}, \\
& B_{j_{1}, \sigma}\left(\theta^{\prime}\right):=\sum_{\ell_{1}=0}^{q_{1}}\left(\sum_{\ell^{\prime}=-q^{\prime}}^{q^{\prime}} B_{\ell_{1}, \ell^{\prime}, j_{1}, \sigma} \mathrm{e}^{i \ell^{\prime} \cdot \theta^{\prime}}\right) T^{\ell_{1}} .
\end{aligned}
$$


This reduces the system to a collection of one-dimensional problems parametrized by the frequencies $\xi^{\prime}$. The end of the proof is based on the following observation:

Lemma 3.1. Let Assumptions 3.1 and 3.2 be satisfied, and assume that the scheme (31) is strongly stable in the sense of Definition [3.1. Then for all $\xi^{\prime} \in \mathbb{R}^{d-1}$, the scheme (35) satisfies Assumptions 2.1 and 2.2, and is strongly stable in the sense of Definition 2.1. Moreover, the estimate of Theorem 2.1 holds with a constant $C$ that is independent of $\xi^{\prime}$.

Instead of turning directly to the proof of Lemma 3.1. we first complete the proof of Theorem 3.1. By Lemma 3.1, the scheme (35) satisfies the assumptions of Theorem 2.1, and the constant $C$ in (6) is independent of $\xi^{\prime}$. Hence,

$$
\begin{aligned}
& \sup _{n \geq 0} \mathrm{e}^{-2 \gamma n \Delta t}\left\|\widehat{V^{n}}\left(\xi^{\prime}\right)\right\|_{1-r_{1},+\infty}^{2}+\frac{\gamma}{\gamma \Delta t+1} \sum_{n \geq 0} \Delta t \mathrm{e}^{-2 \gamma n \Delta t}\left\|\widehat{V^{n}}\left(\xi^{\prime}\right)\right\|_{1-r_{1},+\infty}^{2} \\
& +\sum_{n \geq 0} \Delta t \mathrm{e}^{-2 \gamma n \Delta t} \sum_{j_{1}=1-r_{1}}^{0}\left|\widehat{V_{j_{1}}^{n}}\left(\xi^{\prime}\right)\right|^{2} \leq C\left\{\sum_{n \geq 1} \Delta t \mathrm{e}^{-2 \gamma n \Delta t} \sum_{j_{1}=1-r_{1}}^{0}\left|\widehat{g_{j_{1}}^{n}}\left(\xi^{\prime}\right)\right|^{2}\right. \\
& \left.+\left\|\widehat{f}\left(\xi^{\prime}\right)\right\|_{1-r,+\infty}^{2}+\frac{\gamma \Delta t+1}{\gamma} \sum_{n \geq 0} \Delta t \mathrm{e}^{-2 \gamma(n+1) \Delta t}\left\|\widehat{F^{n}}\left(\xi^{\prime}\right)\right\|_{1,+\infty}^{2}\right\} .
\end{aligned}
$$

For the first term on the left-hand side of (34), we appeal to the Fubini and the Plancherel theorems to get

$$
\begin{aligned}
\sup _{n \geq 0} \mathrm{e}^{-2 \gamma n \Delta t}\left\|U^{n}\right\|_{1-r_{1},+\infty}^{2} & =\sup _{n \geq 0} \mathrm{e}^{-2 \gamma n \Delta t} \int_{\mathbb{R}^{d-1}}\left\|V^{n}\left(x^{\prime}\right)\right\|_{1-r_{1},+\infty}^{2} \mathrm{~d} x^{\prime} \\
& =\frac{1}{(2 \pi)^{d-1}} \sup _{n \geq 0} \mathrm{e}^{-2 \gamma n \Delta t} \int_{\mathbb{R}^{d-1}}\left\|\widehat{V^{n}}\left(\xi^{\prime}\right)\right\|_{1-r_{1},+\infty}^{2} \mathrm{~d} \xi^{\prime} \\
& \leq \frac{1}{(2 \pi)^{d-1}} \int_{\mathbb{R}^{d-1}} \operatorname{sup~}_{n \geq 0} \mathrm{e}^{-2 \gamma n \Delta t}\left\|\widehat{V^{n}}\left(\xi^{\prime}\right)\right\|_{1-r_{1},+\infty}^{2} \mathrm{~d} \xi^{\prime},
\end{aligned}
$$

after switching the order of the supremum and of the integral. For the other terms on the left-hand side of (34) we just note, proceeding as above, that

$$
\begin{aligned}
& \frac{\gamma}{\gamma \Delta t+1} \sum_{n \geq 0} \Delta t \mathrm{e}^{-2 \gamma n \Delta t}\left\|U^{n}\right\|_{1-r_{1},+\infty}^{2}+\sum_{n \geq 0} \Delta t \mathrm{e}^{-2 \gamma n \Delta t} \sum_{j_{1}=1-r_{1}}^{0}\left\|U_{j_{1},}^{n}\right\|^{2} \\
& =\int_{\mathbb{R}^{d-1}}\left(\frac{\gamma}{\gamma \Delta t+1} \sum_{n \geq 0} \Delta t \mathrm{e}^{-2 \gamma n \Delta t}\left\|V^{n}\left(x^{\prime}\right)\right\|_{1-r_{1},+\infty}^{2}\right. \\
& \left.+\sum_{n \geq 0} \Delta t \mathrm{e}^{-2 \gamma n \Delta t} \sum_{j_{1}=1-r_{1}}^{0}\left|V_{j_{1}}^{n}\left(x^{\prime}\right)\right|^{2}\right) \mathrm{d} x^{\prime} \\
& =\int_{\mathbb{R}^{d-1}}\left(\frac{\gamma}{\gamma \Delta t+1} \sum_{n \geq 0} \Delta t \mathrm{e}^{-2 \gamma n \Delta t}\left\|\widehat{V^{n}}\left(\xi^{\prime}\right)\right\|_{1-r_{1},+\infty}^{2}\right. \\
& \left.+\sum_{n \geq 0} \Delta t \mathrm{e}^{-2 \gamma n \Delta t} \sum_{j_{1}=1-r_{1}}^{0}\left|\widehat{V_{j_{1}}^{n}}\left(\xi^{\prime}\right)\right|^{2}\right) \frac{\mathrm{d} \xi^{\prime}}{(2 \pi)^{d-1}} .
\end{aligned}
$$


The combination of the last two estimates with (36) proves (34). Observe it is crucial that the right-hand side of (6) only contains $\ell^{2}$ type norms so that we may apply Plancherel's Theorem, whereas the $\ell^{\infty}$-norm only appears on the left-hand side of (6). This concludes the proof of Theorem 3.1 .

We turn to the proof of Lemma 3.1

Proof of Lemma 3.1. In (35), the coefficients of the operators $Q\left(\theta^{\prime}\right), B_{j,-1}\left(\theta^{\prime}\right)$, $B_{j, 0}\left(\theta^{\prime}\right)$ are complex matrices. Our proof of Theorem 2.1 was basically written for real matrices. However, the proofs of the results in Section 2 are unchanged when complex matrices are considered provided Assumptions 2.1 and 2.2 are satisfied. In particular, Assumption 2.2 should hold for all sequences $v \in \ell^{2}$ taking values in $\mathbb{C}^{D}$. Only slight modifications are needed in order to consider complex matrices: use Hermitian forms instead of real quadratic forms, Hermitian matrices instead of real symmetric matrices and so on. From now on, we directly apply the results of Section 2 in the more general framework of complex matrices.

Let us first show that Assumption 2.2 is satisfied. We know that the norm of $Q$ as an operator on $\ell^{2}\left(\mathbb{Z}^{d}\right)$ is not greater than 1 . Using Plancherel's Theorem, this property is equivalent to the fact that the norm of the symbol $\widehat{Q}$ of $Q$ is not greater than 1. Decomposing frequencies $\theta \in \mathbb{R}^{d}$ as $\theta=\left(\theta_{1}, \theta^{\prime}\right)$, the symbol or amplification matrix $\widehat{Q}(\theta)$ is given by

$$
\widehat{Q}(\theta)=\sum_{\ell_{1}=-r_{1}}^{p_{1}}\left(\sum_{\ell^{\prime}=-r^{\prime}}^{p^{\prime}} A_{\ell_{1}, \ell^{\prime}} \mathrm{e}^{i \ell^{\prime} \cdot \theta^{\prime}}\right) \mathrm{e}^{i \ell_{1} \theta_{1}} .
$$

In other words, the symbol of the operator $Q\left(\theta^{\prime}\right)$ is nothing more than $\widehat{Q}\left(\cdot, \theta^{\prime}\right)$. This shows that, for all $\theta^{\prime}$, the norm of $Q\left(\theta^{\prime}\right)$ as an operator on $\ell^{2}(\mathbb{Z})$ is not larger than 1. Assumption 2.2 is thus satisfied.

Let us turn to Assumption 2.1. With slight abuse of notation, if $w \in \ell^{2}\left(\mathbb{Z}^{d-1}\right)$, we still denote $w$ the piecewise constant function defined on $\mathbb{R}^{d-1}$, and whose value on the mesh element with index $j^{\prime}$ is $w_{j^{\prime}}$. Using Plancherel's Theorem, we have

$$
\begin{aligned}
\left\|\mathbb{A}_{p_{1}}(z) w\right\|^{2} & =\frac{1}{(2 \pi)^{d-1}} \int_{\mathbb{R}^{d-1}}\left|\left(\widehat{\mathbb{A}_{p_{1}}(z)} w\right)\left(\xi^{\prime}\right)\right|^{2} \mathrm{~d} \xi^{\prime} \\
& =\frac{1}{(2 \pi)^{d-1}} \int_{\mathbb{R}^{d-1}}\left|\mathbb{A}_{p_{1}}\left(z, \theta^{\prime}\right) \widehat{w}\left(\xi^{\prime}\right)\right|^{2} \mathrm{~d} \xi^{\prime},
\end{aligned}
$$

where $\theta^{\prime}$ is again a shorthand notation for $\left(\xi_{2} \Delta x_{2}, \ldots, \xi_{d} \Delta x_{d}\right)$, and where the matrices $\mathbb{A}_{p_{1}}\left(z, \theta^{\prime}\right)$ are defined by

$$
\mathbb{A}_{p_{1}}\left(z, \theta^{\prime}\right):=\delta_{p_{1} 0} I-\frac{1}{z} \sum_{\ell^{\prime}=-r^{\prime}}^{p^{\prime}} A_{p_{1}, \ell^{\prime}} \mathrm{e}^{i \ell^{\prime} \cdot \theta^{\prime}} ;
$$

see (33). The operator $\mathbb{A}_{p_{1}}$ is coercive if and only if the matrices $\mathbb{A}_{p_{1}}\left(z, \theta^{\prime}\right)$ are invertible for all $z \in \mathbb{C}$ with $|z| \geq 1$ and all $\theta^{\prime} \in \mathbb{R}^{d-1}$. This proves that Assumption 2.1 is satisfied for all $\theta^{\prime} \in \mathbb{R}^{d-1}$.

Next we argue that the constants in Theorem 2.1 are independent of $\xi^{\prime}$. A close look at the proof shows that the only places where the constants may depend on $\xi^{\prime}$ are Lemma 2.2 and (23). On the one hand, Assumption 3.1 provides a lower bound for the coercivity constants of $\mathbb{A}_{p_{1}}\left(z, \theta^{\prime}\right)$ which is uniform in $\theta^{\prime}$, so that the constant in Lemma 2.2 is uniform in $\theta^{\prime}$. On the other hand, for $p_{1}=0$, we claim that the 
norm $|\cdot|_{H\left(\theta^{\prime}\right)}$ and the standard Hermitian norm $|\cdot|$ are equivalent uniformly in $\theta^{\prime}$ so that the constant in (23) can be chosen independent of $\theta^{\prime}$. To this aim, it is enough to prove that

$$
\begin{aligned}
\sup _{\theta^{\prime} \in \mathbb{R}^{d-1}}\left|\mathbb{A}_{0}\left(1, \theta^{\prime}\right)\right| & <\infty, \\
\sup _{\theta^{\prime} \in \mathbb{R}^{d-1}} \rho\left(\mathbb{A}_{0}\left(1, \theta^{\prime}\right)\right) & <1 .
\end{aligned}
$$

Since $\theta^{\prime} \mapsto \mathbb{A}_{0}\left(1, \theta^{\prime}\right)$ is continuous and periodic on $\mathbb{R}^{d-1}$, (38) is trivial, the supremum in (39) is attained and the bound follows from Assumption 3.1 and (37).

The only remaining task is to prove that the scheme (35) is strongly stable in the sense of Definition 2.1. This is done, as above, by applying Plancherel's Theorem to the strong stability estimate in Definition 3.1. We omit the details and refer to 11 for a similar analysis.

3.2. A multidimensional version of the Goldberg-Tadmor Lemma. The same arguments as above (Fourier transform in the tangential variables and application of the one-dimensional results of Section 21) also apply to the case of non-homogeneous Dirichlet boundary conditions. More precisely, let us consider the following auxiliary problem with Dirichlet boundary conditions:

$$
\begin{cases}V_{j_{1}, j^{\prime}}^{n+1}=Q V_{j_{1}, j^{\prime}}^{n}+\Delta t F_{j_{1}, j^{\prime}}^{n}, & j_{1} \geq 1, j^{\prime} \in \mathbb{Z}^{d-1}, \quad n \geq 0, \\ V_{j_{1}, j^{\prime}}^{n+1}=g_{j_{1}, j^{\prime}}^{n+1}, & j_{1}=1-r_{1}, \ldots, 0, j^{\prime} \in \mathbb{Z}^{d-1}, \quad n \geq 0, \\ V_{j_{1}, j^{\prime}}^{0}=f_{j_{1}, j^{\prime}}, & j_{1} \geq 1-r_{1}, j^{\prime} \in \mathbb{Z}^{d-1} .\end{cases}
$$

The multidimensional version of Theorem 2.2 is

Theorem 3.2. Let Assumptions 3.1 and 3.2 be satisfied. Then there exists a constant $C$ such that for all $\gamma>0$ and all $\Delta t \in] 0,1]$, the solution $V$ to (40) satisfies the estimate

$$
\begin{aligned}
\sup _{n \geq 0} \mathrm{e}^{-2 \gamma n \Delta t}\left\|V^{n}\right\|_{1-r_{1},+\infty}^{2}+\frac{\gamma}{\gamma \Delta t+1} \sum_{n \geq 0} \Delta t \mathrm{e}^{-2 \gamma n \Delta t}\left\|V^{n}\right\|_{1-r_{1},+\infty}^{2} \\
+\sum_{n \geq 0} \Delta t \mathrm{e}^{-2 \gamma n \Delta t} \sum_{j_{1}=1-r_{1}}^{\max \left(p_{1}, q_{1}+1\right)}\left\|V_{j_{1},}^{n} .\right\|^{2} \leq C\left\{\sum_{n \geq 1} \Delta t \mathrm{e}^{-2 \gamma n \Delta t} \sum_{j_{1}=1-r_{1}}^{0}\left\|g_{j_{1},}^{n}\right\|^{2}\right. \\
\left.+\|f\|_{1-r_{1},+\infty}^{2}+\frac{\gamma \Delta t+1}{\gamma} \sum_{n \geq 0} \Delta t \mathrm{e}^{-2 \gamma(n+1) \Delta t}\left\|F^{n}\right\|_{1,+\infty}^{2}\right\} .
\end{aligned}
$$

In particular, the discretization (40) is strongly stable in the sense of Definition 3.1 .

We do not detail the proof of Theorem 3.2 since the arguments are similar to the arguments used in the proof of Theorem 3.1 for passing from one-dimensional results to multidimensional results.

\section{Multidimensional problems: Variable coefficients}

When dealing with variable coefficients, the natural functional setting is $L^{2}\left(\mathbb{R}^{d}\right)$ instead of $\ell^{2}\left(\mathbb{Z}^{d}\right)$ and we need to adapt the definition of norms as follows. Let $\Delta x_{i}>0$ for $i=1, \ldots, d$ be $d$ space steps. For all integers $m_{1} \leq m_{2}$, we let

$$
\|u\|_{m_{1}, m_{2}}^{2}:=\int_{\left[m_{1} \Delta x_{1},\left(m_{2}+1\right) \Delta x_{1}\left[\times \mathbb{R}^{d-1}\right.\right.}\left|u\left(x_{1}, x^{\prime}\right)\right|^{2} \mathrm{~d} x_{1} \mathrm{~d} x^{\prime}
$$


denote the $L^{2}$-norm on the set $\left[m_{1} \Delta x_{1},\left(m_{2}+1\right) \Delta x_{1}\left[\times \mathbb{R}^{d-1}\left(m_{1}\right.\right.\right.$ may equal $-\infty$ and $m_{2}$ may equal $\left.+\infty\right)$. Note that this definition is consistant with the discrete underlying structure. This explains why we integrate up to $\left(m_{2}+1\right) \Delta x_{1}$ and not $m_{2} \Delta x_{1}$. The corresponding scalar product is $(\cdot, \cdot)_{m_{1}, m_{2}}$.

We will also make use of the $L^{2}\left(\left[0, \Delta x_{1}\left[\times \mathbb{R}^{d-1}\right)\right.\right.$-norm that we denote by $\|\cdot\|$ : for all $v \in L^{2}\left(\left[0, \Delta x_{1}\left[\times \mathbb{R}^{d-1}\right)\right.\right.$,

$$
\|v\|^{2}:=\int_{\left[0, \Delta x_{1}\left[\times \mathbb{R}^{d-1}\right.\right.}\left|v\left(x_{1}, x^{\prime}\right)\right|^{2} \mathrm{~d} x_{1} \mathrm{~d} x^{\prime} .
$$

The associated scalar product is $\left\langle\cdot ; \cdot \cdot\right.$. For convenience, for all $j_{1} \in \mathbb{Z}$ and all $v \in L^{2}\left(\left[j_{1} \Delta x_{1},\left(j_{1}+1\right) \Delta x_{1}\left[\times \mathbb{R}^{d-1}\right)\right.\right.$, we also set

$$
\|v\|_{j_{1}}^{2}:=\int_{\left[j_{1} \Delta x_{1},\left(j_{1}+1\right) \Delta x_{1}\left[\times \mathbb{R}^{d-1}\right.\right.}\left|v\left(x_{1}, x^{\prime}\right)\right|^{2} \mathrm{~d} x_{1} \mathrm{~d} x^{\prime},
$$

whose associated scalar product is $\langle\cdot ; \cdot\rangle_{j_{1}}$.

4.1. Main result for variable coefficients. We now consider the hyperbolic initial boundary value problem (29) and further assume that the matrices $A_{1}, \ldots$, $A_{d}, B$ may depend on time and space. The one-step finite difference approximation (31) is replaced by

$$
\left\{\begin{array}{rlrl}
U^{n+1}(x)= & Q^{n}(x) U^{n}(x)+\Delta t F^{n}(x), & & x_{1} \geq \Delta x_{1}, \quad n \geq 0 \\
U^{n+1}(x)= & B_{-1}^{n}(x) U^{n+1}(x) & & \\
& +B_{0}^{n}(x) U^{n}(x)+g^{n+1}(x), & & \left(1-r_{1}\right) \Delta x_{1} \leq x_{1} \leq \Delta x_{1}, n \geq 0 \\
U^{0}(x)=f(x), & & x_{1} \geq\left(1-r_{1}\right) \Delta x_{1}
\end{array}\right.
$$

where the operators $Q^{n}$ and $B_{\sigma}^{n}$ may now depend on the time step $n$ and the space variable $x$. In particular, we have

$$
\begin{aligned}
Q^{n}(x) & :=\sum_{\ell_{1}=-r_{1}}^{p_{1}}\left(\sum_{\ell^{\prime}=-r^{\prime}}^{p^{\prime}} A_{\ell_{1}, \ell^{\prime}}^{n}(x) T_{\Delta x^{\prime}}^{\prime}{ }^{\ell^{\prime}}\right) T_{\Delta x_{1}}{ }^{\ell_{1}}, \\
B_{\sigma}^{n}(x) & :=\sum_{\ell_{1}=0}^{q_{1}}\left(\sum_{\ell^{\prime}=-q^{\prime}}^{q^{\prime}} B_{\ell_{1}, \ell^{\prime}, \sigma}^{n}(x) T_{\Delta x^{\prime}}^{\prime}{ }^{\ell^{\prime}}\right) T_{\Delta x_{1}}{ }^{\ell_{1}},
\end{aligned}
$$

where $T_{\Delta x_{1}}$ and $T_{\Delta x^{\prime}}^{\prime}$ are the shift operators associated with $\Delta x:=\left(\Delta x_{1}, \Delta x^{\prime}\right)$ :

$$
T_{\Delta x_{1}} U(x):=U\left(x_{1}+\Delta x_{1}, x^{\prime}\right), \quad T_{\Delta x^{\prime}}^{\prime} U(x):=U\left(x_{1}, x^{\prime}+\Delta x^{\prime}\right),
$$

for all $U \in L^{2}\left(\left[a,+\infty\left[\times \mathbb{R}^{d-1}\right), a \in \mathbb{R}\right.\right.$. In (43), the integers $p_{1}, r_{1}, q_{1}, p^{\prime}, r^{\prime}, q^{\prime}$ do not depend on $n$ nor on the space variable $x$. Moreover, the matrices $A_{\ell}^{n}, B_{\ell, \sigma}^{n}$ are as follows:

$$
A_{\ell}^{n}: \mathbb{R}^{d} \longrightarrow \mathcal{M}_{D}(\mathbb{R}), \quad B_{\ell, \sigma}^{n}:\left[\left(1-r_{1}\right) \Delta x_{1}, \Delta x_{1}\left[\times \mathbb{R}^{d-1} \longrightarrow \mathcal{M}_{D}(\mathbb{R}) .\right.\right.
$$

The time step $\Delta t \in] 0,1]$ is kept as a small parameter, while the space steps $\Delta x_{i}$ are given by $\Delta t=\lambda_{i} \Delta x_{i}$ where the $\lambda_{i}$ 's are fixed positive numbers. The generalization of stability to the variable coefficients case is:

Definition 4.1 (Strong stability [11]). The finite difference approximation (42) is said to be strongly stable if there exist $C>0$ and $\bar{\gamma}>0$ such that for all $\gamma \geq \bar{\gamma}$ 
and all $\Delta t \in] 0,1]$, the solution $U$ of (42) with $f=0$ satisfies the estimate

$$
\begin{aligned}
& \frac{\gamma}{\gamma \Delta t+1} \sum_{n \geq 0} \Delta t \mathrm{e}^{-2 \gamma n \Delta t}\left\|U^{n}\right\|_{1-r_{1},+\infty}^{2}+\sum_{n \geq 0} \Delta t \mathrm{e}^{-2 \gamma n \Delta t} \sum_{j_{1}=1-r_{1}}^{0} \frac{\left\|U^{n}\right\|_{j_{1}}^{2}}{\Delta x_{1}} \\
& \leq C\left\{\frac{\gamma \Delta t+1}{\gamma} \sum_{n \geq 0} \Delta t \mathrm{e}^{-2 \gamma(n+1) \Delta t}\left\|F^{n}\right\|_{1,+\infty}^{2}\right. \\
&\left.+\sum_{n \geq 1} \Delta t \mathrm{e}^{-2 \gamma n \Delta t} \sum_{j_{1}=1-r_{1}}^{0} \frac{\left\|g^{n}\right\|_{j_{1}}^{2}}{\Delta x_{1}}\right\} .
\end{aligned}
$$

Observe that in Definition 4.1, the boundary norms $\|\cdot\|_{j_{1}}$ are divided by $\Delta x_{1}$ : they represent mean values on the intervals $\left[j_{1} \Delta x_{1},\left(j_{1}+1\right) \Delta x_{1}[\right.$. In the limit $\Delta x_{1} \rightarrow 0$, those mean values tend to $L^{2}$-norms for traces on the boundary $\left\{x_{1}=0\right\}$.

Let us now generalize Assumptions 3.1 and 3.2 to the case of variable coefficients. For all $n \geq 0, m \in \mathbb{Z}, \ell_{1}=-r_{1}, \ldots, p_{1}$, and $z \in \mathbb{C} \backslash\{0\}$, let us define the linear mappings $\mathbb{A}_{\ell_{1}, m}^{n}(z)$ on $L^{2}\left(\left[0, \Delta x_{1}\left[\times \mathbb{R}^{d-1}\right)\right.\right.$ by the formula

$$
\mathbb{A}_{\ell_{1}, m}^{n}(z) w(x):=\delta_{\ell_{1} 0} w(x)-\frac{1}{z} \sum_{\ell^{\prime}=-r^{\prime}}^{p^{\prime}} A_{\ell_{1}, \ell^{\prime}}^{n}\left(x_{1}+m \Delta x_{1}, x^{\prime}\right) T_{\Delta x^{\prime}}^{\prime} \ell^{\prime} w(x) .
$$

We make the following assumption:

Assumption 4.1. The mappings $\mathbb{A}_{p_{1}, m}^{n}(z)$ are coercive on $L^{2}\left(\left[0, \Delta x_{1}\left[\times \mathbb{R}^{d-1}\right)\right.\right.$ uniformly in $m \in \mathbb{Z}$ and $n \geq 0$, for all $z \in \mathbb{C}$ with $|z| \geq 1$. More precisely, there exists a constant $c>0$ such that for all $z \in \mathbb{C}$ with $|z| \geq 1$, for all $m \in \mathbb{Z}$ and $n \in \mathbb{N}$, we have

$$
\forall w \in L^{2}\left(\left[0, \Delta x_{1}\left[\times \mathbb{R}^{d-1}\right), \quad\left\|\mathbb{A}_{p_{1}, m}^{n}(z) w\right\| \geq \frac{c}{|z|^{\nu}}\|w\|, \quad \nu:= \begin{cases}1 & \text { if } p_{1}>0, \\ 0 & \text { otherwise } .\end{cases}\right.\right.
$$

For the second assumption, we use the following pointwise version of Assumption 3.2 .

Assumption 4.2. For all $n \geq 0$ and $x \in \mathbb{R}^{d}$, the symbol of $Q^{n}$ is bounded in the sense

$$
\forall \xi \in \mathbb{R}^{d}, \quad\left|\sum_{\ell=-r}^{p} \mathrm{e}^{i \xi \cdot \ell} A_{\ell}^{n}(x)\right| \leq 1 .
$$

The last assumption (regularity of the coefficients) will allow us to deduce that $Q^{n}$ does not increase the energy up to an error of order $|\Delta x|$, using the pseudodifference calculus developed by Lax and Nirenberg; see [10, Corollary 1.1].

Assumption 4.3. The coefficients in (43) satisfy

$$
A_{\ell}^{n} \in \mathcal{C}_{b}^{2}\left(\mathbb{R}^{d}\right), \quad B_{\ell, \sigma}^{n} \in L^{\infty}\left(\left[\left(1-r_{1}\right) \Delta x_{1}, \Delta x_{1}\left[\times \mathbb{R}^{d-1}\right)\right.\right.
$$

uniformly in $n \in \mathbb{N}, \ell$ and $\sigma$, with $\mathcal{C}_{b}^{2}\left(\mathbb{R}^{d}\right)$ the space of bounded $\mathcal{C}^{2}$ functions with bounded first and second derivatives.

In particular, we have 
Lemma 4.1 (Sharp Gårding's inequality [10]). Let the operators $Q^{n}, n \in \mathbb{N}$, satisfy Assumptions 4.2 and 4.3 . Then there exists a constant $C>0$ that does not depend on $n$ nor on $\Delta x$ such that for all $w \in L^{2}\left(\mathbb{R}^{d}\right)$ and $n \geq 0$, we have

$$
\left\|Q^{n} w\right\|_{-\infty,+\infty}^{2} \leq(1+C|\Delta x|)\|w\|_{-\infty,+\infty}^{2} .
$$

Our main result corresponding to Theorem 3.1 is then

Theorem 4.1. Let Assumptions 4.1, 4.2 and 4.3 be satisfied, and assume that the scheme (42) is strongly stable in the sense of Definition 4.1, Then there exist $C>0$ and $\bar{\gamma}>0$ such that for all $\gamma \geq \bar{\gamma}$ and all $\Delta t \in] 0,1]$, the solution $U$ to (42) satisfies the estimate

$$
\begin{aligned}
& \sup _{n \geq 0} \mathrm{e}^{-2 \gamma n \Delta t}\left\|U^{n}\right\|_{1-r_{1},+\infty}^{2}+\frac{\gamma}{\gamma \Delta t+1} \sum_{n \geq 0} \Delta t \mathrm{e}^{-2 \gamma n \Delta t}\left\|U^{n}\right\|_{1-r_{1},+\infty}^{2} \\
&+\sum_{n \geq 0} \Delta t \mathrm{e}^{-2 \gamma n \Delta t} \sum_{j_{1}=1-r_{1}}^{0} \frac{\left\|U^{n}\right\|_{j_{1}}^{2}}{\Delta x_{1}} \leq C\left\{\frac{\gamma \Delta t+1}{\gamma} \sum_{n \geq 0} \Delta t \mathrm{e}^{-2 \gamma(n+1) \Delta t}\left\|F^{n}\right\|_{1,+\infty}^{2}\right. \\
&\left.+\|f\|_{1-r_{1},+\infty}^{2}+\sum_{n \geq 1} \Delta t \mathrm{e}^{-2 \gamma n \Delta t} \sum_{j_{1}=1-r_{1}}^{0} \frac{\left\|g^{n}\right\|_{j_{1}}^{2}}{\Delta x_{1}}\right\}
\end{aligned}
$$

As for the one-dimensional case, our proof of Theorem 4.1 relies only on the energy method.

4.2. A version of the Goldberg-Tadmor Lemma for variable coefficients. We consider the auxiliary discretization corresponding to (40) in the variable coefficients case, where the Dirichlet conditions are enforced at the boundary:

$$
\begin{cases}V^{n+1}(x)=Q^{n}(x) V^{n}(x)+\Delta t F^{n}(x), & x \in\left[\Delta x_{1},+\infty\left[\times \mathbb{R}^{d-1}, \quad n \geq 0,\right.\right. \\ V^{n+1}(x)=g^{n+1}(x), & x \in\left[\left(1-r_{1}\right) \Delta x_{1}, \Delta x_{1}\left[\times \mathbb{R}^{d-1}, \quad n \geq 0,\right.\right. \\ V^{0}(x)=f(x), & x \in\left[\left(1-r_{1}\right) \Delta x_{1},+\infty\left[\times \mathbb{R}^{d-1} .\right.\right.\end{cases}
$$

The aim of this paragraph is to generalize Theorem 3.2 to the variable coefficients case:

Theorem 4.2. Let Assumptions 4.1, 4.2 and 4.3 be satisfied. Then there exist $C>0$ and $\bar{\gamma}>0$ such that for all $\gamma \geq \bar{\gamma}$ and all $\Delta t \in] 0,1]$, the solution $V$ to (45) satisfies the estimate

$$
\begin{gathered}
\sup _{n \geq 0} \mathrm{e}^{-2 \gamma n \Delta t}\left\|V^{n}\right\|_{1-r_{1},+\infty}^{2}+\frac{\gamma}{\gamma \Delta t+1} \sum_{n \geq 0} \Delta t \mathrm{e}^{-2 \gamma n \Delta t}\left\|V^{n}\right\|_{1-r_{1},+\infty}^{2} \\
+\sum_{n \geq 0} \Delta t \mathrm{e}^{-2 \gamma n \Delta t} \sum_{j_{1}=1-r_{1}}^{\max \left(p_{1}, q_{1}+1\right)} \frac{\left\|V^{n}\right\|_{j_{1}}^{2}}{\Delta x_{1}} \leq C\left\{\|f\|_{1-r_{1},+\infty}^{2}\right. \\
\left.+\frac{\gamma \Delta t+1}{\gamma} \sum_{n \geq 0} \Delta t \mathrm{e}^{-2 \gamma(n+1) \Delta t}\left\|F^{n}\right\|_{1,+\infty}^{2}+\sum_{n \geq 1} \Delta t \mathrm{e}^{-2 \gamma n \Delta t} \sum_{j_{1}=1-r_{1}}^{0} \frac{\left\|g^{n}\right\|_{j_{1}}^{2}}{\Delta x_{1}}\right\} .
\end{gathered}
$$

In particular, the discretization (45) is strongly stable in the sense of Definition 4.1. 
As for Theorem 2.2, the key point in the proof of Theorem 4.2 is the estimate of Lemma 4.1, which is a consequence of Assumptions 4.2 and 4.3. The proof of Theorem 4.2 is similar to the one-dimensional case. Whereas we state precisely all the intermediate results, we only detail the arguments when the space dimension or the new $\mathcal{O}(\Delta x)$ correcting term in Gårding's inequality come into play. As in Section 2, we first prove (46) in the case $F=0$ (no source term in the interior equation), and then in the case $(f, g)=0$ (zero initial data, and homogeneous boundary conditions).

Lemma 4.2. Let Assumptions 4.1, 4.2 and 4.3 be satisfied. Then there exist $C>0$ and $\bar{\gamma}>0$ such that for all $\gamma \geq \bar{\gamma}$ and all $\Delta t \in] 0,1]$, the solution $V$ to (45) with $F=0$ satisfies the estimate

$$
\begin{aligned}
& \mathrm{e}^{2 \gamma \Delta t} \sup _{n \geq 1} \mathrm{e}^{-2 \gamma n \Delta t}\left\|V^{n}\right\|_{1,+\infty}^{2}+\gamma \sum_{n \geq 1} \Delta t \mathrm{e}^{-2 \gamma n \Delta t}\left\|V^{n}\right\|_{1,+\infty}^{2} \\
& \quad+\sum_{n \geq 0} \Delta t \mathrm{e}^{-2 \gamma n \Delta t} \sum_{j_{1}=1-r_{1}}^{p_{1}} \frac{\left\|V^{n}\right\|_{j_{1}}^{2}}{\Delta x_{1}} \leq C\left\{\|f\|_{1-r_{1},+\infty}^{2}\right. \\
& \left.+\sum_{n \geq 1} \Delta t \mathrm{e}^{-2 \gamma n \Delta t} \sum_{j_{1}=1-r_{1}}^{0} \frac{\left\|g^{n}\right\|_{j_{1}}^{2}}{\Delta x_{1}}\right\}
\end{aligned}
$$

Proof of Lemma 4.2. We decompose the operator $Q^{n}$ as $Q^{n}=I+\widetilde{Q}^{n}$, and introduce the function $W \in L^{2}\left(\mathbb{R}^{d}\right)$ defined by $W(x):=V^{n}(x)$ for $x_{1} \geq\left(1-r_{1}\right) \Delta x_{1}$ and $W(x):=0$ otherwise. We apply Gårding's inequality to $Q^{n} W$, obtaining

$$
2\left(W, \widetilde{Q}^{n}\right)_{-\infty, \infty}+\left\|\widetilde{Q}^{n} W\right\|_{-\infty,+\infty}^{2} \leq C|\Delta x|\|W\|_{-\infty,+\infty}^{2} .
$$

Using the definition of $W$, and recalling that

$$
\widetilde{Q}^{n} W(x)= \begin{cases}0 & \text { if } x_{1}<\left(1-r_{1}-p_{1}\right) \Delta x_{1}, \\ \widetilde{Q}^{n} V^{n}(x) & \text { if } x_{1} \geq \Delta x_{1}\end{cases}
$$

this yields an estimate analogous to (13):

$$
\begin{array}{r}
\left\|V^{n+1}\right\|_{1,+\infty}^{2}-\left\|V^{n}\right\|_{1,+\infty}^{2}+\left\|\widetilde{Q}^{n} W\right\|_{1-r_{1}-p_{1},-r_{1}}^{2}+\left\|V^{n}+\widetilde{Q}^{n} W\right\|_{1-r_{1}, 0}^{2} \\
\leq\left\|V^{n}\right\|_{1-r_{1}, 0}^{2}+C \Delta t\left\|V^{n}\right\|_{1-r_{1},+\infty}^{2},
\end{array}
$$

where the constant $C$ does not depend on $n$.

For $p_{1}=0$, the proof goes as for the one-dimensional case and we focus here on the case $p_{1} \geq 1$, for which we need a multidimensional version of Lemma 2.2. Assumption 4.1 yields the following:

Lemma 4.3. Let $p_{1} \geq 1$ and let Assumption 4.1 be satisfied. Then there exists a constant $c>0$ that does not depend on $n \in \mathbb{N}, \Delta t \in] 0,1]$ nor on $V^{n}$ such that the following estimate holds:

$$
\left\|\widetilde{Q}^{n} W\right\|_{1-r_{1}-p_{1},-r_{1}}^{2}+\left\|V^{n}+\widetilde{Q}^{n} W\right\|_{1-r_{1}, 0}^{2} \geq c\left\|V^{n}\right\|_{1-r_{1}, p_{1}}^{2} .
$$


Using Lemma 4.3, (48) then becomes

$$
\begin{array}{r}
\left\|V^{n+1}\right\|_{1,+\infty}^{2}-\left\|V^{n}\right\|_{1,+\infty}^{2}+c \sum_{j_{1}=1-r_{1}}^{p_{1}}\left\|V^{n}\right\|_{j_{1}}^{2} \\
\leq \sum_{j_{1}=1-r_{1}}^{0}\left\|V^{n}\right\|_{j_{1}}^{2}+C \Delta t\left\|V^{n}\right\|_{1-r_{1},+\infty}^{2} .
\end{array}
$$

For $\gamma>0$, introducing the notation

$$
\begin{aligned}
& \mathcal{V}_{n}:=\mathrm{e}^{-2 \gamma n \Delta t}\left\|V^{n}\right\|_{1,+\infty}^{2}, \\
& \mathcal{B}_{n}:=\mathrm{e}^{-2 \gamma n \Delta t} \sum_{j_{1}=1-r_{1}}^{p_{1}} \frac{\left\|V^{n}\right\|_{j_{1}}^{2}}{\Delta x_{1}}, \\
& \mathcal{G}_{n}:=\mathrm{e}^{-2 \gamma n \Delta t} \sum_{j_{1}=1-r_{1}}^{0} \frac{\left\|V^{n}\right\|_{j_{1}}^{2}}{\Delta x_{1}},
\end{aligned}
$$

estimate (50), multiplied by $\mathrm{e}^{-2 \gamma n \Delta t}$, becomes

$$
\mathrm{e}^{2 \gamma \Delta t} \mathcal{V}_{n+1}-\mathcal{V}_{n}+\frac{c}{\lambda_{1}} \Delta t \mathcal{B}_{n} \leq \frac{1}{\lambda_{1}} \Delta t \mathcal{G}_{n}+C \Delta t\left(\mathcal{V}_{n}+\frac{1}{\lambda_{1}} \Delta t \mathcal{G}_{n}\right)
$$

Summing for $n$ from 0 to $N$ and using $\Delta t \leq 1$ then yields

$$
\mathrm{e}^{2 \gamma \Delta t} \mathcal{V}_{N+1}+\frac{\mathrm{e}^{2 \gamma \Delta t}-1}{\Delta t} \sum_{n=1}^{N} \Delta t \mathcal{V}_{n}+c \sum_{n=0}^{N} \Delta t \mathcal{B}_{n} \leq \mathcal{V}_{0}+C \sum_{n=0}^{N} \Delta t \mathcal{G}_{n}+C \sum_{n=0}^{N} \Delta t \mathcal{V}_{n}
$$

Since

$$
\frac{\mathrm{e}^{2 \gamma \Delta t}-1}{\Delta t} \geq 2 \gamma \geq \gamma+C
$$

for $\gamma \geq \bar{\gamma}:=C$, we can absorb the last term of the former inequality in the left-hand side to obtain

$$
\begin{aligned}
\mathrm{e}^{2 \gamma \Delta t} \sup _{n \geq 1} \mathcal{V}_{n}+\gamma \sum_{n \geq 1} \Delta t \mathcal{V}_{n}+\sum_{n \geq 0} \Delta t \mathcal{B}_{n} & \leq C\left(\mathcal{V}_{0}+\Delta x_{1} \mathcal{G}_{0}+\sum_{n \geq 1} \Delta t \mathcal{G}_{n}\right) \\
& \leq C\left(\mathcal{V}_{0}+\sum_{n \geq 1} \Delta t \mathcal{G}_{n}\right),
\end{aligned}
$$

noting that $\Delta x_{1} \mathcal{G}_{0} \leq \mathcal{V}_{0}$

It remains to prove Lemma 4.3 .

Proof of Lemma 4.3. As for the proof of Lemma 2.2. we wish to show that a certain qudratic form is positive definite. Note, however, that the quadratic form in Lemma 2.2 acts on a finite-dimensional space, whereas here it acts on the infinitedimensional space $L^{2}\left(\left[0, \Delta x_{1}\left[\times \mathbb{R}^{d-1}\right)\right.\right.$. For this reason, we need the following property.

Let $\mathcal{L}_{1}, \mathcal{L}_{2}, \mathcal{L}: L^{2}\left(\left[0, \Delta x_{1}\left[\times \mathbb{R}^{d-1}\right) \rightarrow L^{2}\left(\left[0, \Delta x_{1}\left[\times \mathbb{R}^{d-1}\right)\right.\right.\right.\right.$ be three continuous linear mappings, and assume in addition that $\mathcal{L}_{1}, \mathcal{L}_{2}$ are coercive: there exists $c>0$ such that for all $v \in L^{2}\left(\left[0, \Delta x_{1}\left[\times \mathbb{R}^{d-1}\right)\right.\right.$, one has $\left\|\mathcal{L}_{1} v\right\| \geq c\|v\|$ and $\left\|\mathcal{L}_{2} v\right\| \geq c\|v\|$. 
Then, there exists some $\tilde{c}>0$ that only depends on $c$ and on the operator norm $\|\mathcal{L}\|$ such that for all $v_{1}, v_{2} \in L^{2}\left(\left[0, \Delta x_{1}\left[\times \mathbb{R}^{d-1}\right)\right.\right.$, it holds that

$$
\left\|\mathcal{L}_{1} v_{1}\right\|^{2}+\left\|\mathcal{L}_{2} v_{2}+\mathcal{L} v_{1}\right\|^{2} \geq \tilde{c}\left(\left\|v_{1}\right\|^{2}+\left\|v_{2}\right\|^{2}\right) .
$$

Before proceeding to the induction argument proper, we rephrase the left-hand side of (49) in order to use the previous abstract argument. To this aim, we define some functions $u_{1}, \ldots, u_{p_{1}+r_{1}} \in L^{2}\left(\left[0, \Delta x_{1}\left[\times \mathbb{R}^{d-1}\right)\right.\right.$ by

$$
u_{1}(x):=V^{n}\left(x_{1}+\left(1-r_{1}\right) \Delta x_{1}, x^{\prime}\right), \ldots, u_{p_{1}+r_{1}}(x):=V^{n}\left(x_{1}+p_{1} \Delta x_{1}, x^{\prime}\right),
$$

so that the left-hand side $I$ of (49) takes the form

$$
\begin{aligned}
I= & \sum_{m=1-r_{1}-p_{1}}^{0} \int_{0}^{\Delta x_{1}} \int_{\mathbb{R}^{d-1}} \mid \mathbb{A}_{p_{1}, m}^{n}(-1) u_{m+p_{1}+r_{1}}(x) \\
& +\left.\sum_{1 \leq l<m+p_{1}+r_{1}} \widetilde{A}_{m, l}^{n}(x) u_{l}(x)\right|^{2} \mathrm{~d} x_{1} \mathrm{~d} x^{\prime}
\end{aligned}
$$

for some uniformly bounded matrices $\widetilde{A}_{m, l}^{n}$.

Let now $\left\{\mathcal{L}_{m, l}\right\}_{1 \leq m \leq p_{1}+r_{1}, 1 \leq l \leq m}$ be some continuous linear mappings on the space $L^{2}\left(\left[0, \Delta x_{1}\left[\times \mathbb{R}^{d-1}\right)\right.\right.$, such that $\left\{\mathcal{L}_{m, 1}\right\}_{1 \leq m \leq p_{1}+r_{1}}$ are uniformly coercive. Then by a straightforward induction argument based on (51), there exists a constant $c_{0}>0$ such that for all $v_{1}, \ldots, v_{p_{1}+r_{1}} \in L^{2}\left(\left[0, \Delta x_{1}\left[\times \mathbb{R}^{d-1}\right)\right.\right.$ one has

$$
\begin{aligned}
\left\|\mathcal{L}_{1,1} v_{1}\right\|^{2}+\left\|\mathcal{L}_{2,1} v_{2}+\mathcal{L}_{2,2} v_{1}\right\|^{2}+\cdots & \\
+\| \mathcal{L}_{p_{1}+r_{1}, 1} v_{p_{1}+r_{1}}+\sum_{l=2}^{p_{1}+r_{1}} \mathcal{L}_{p_{1}+r_{1}, l} & v_{p_{1}+r_{1}+1-l} \|^{2} \\
& \geq c_{0} \sum_{m=1}^{p_{1}+r_{1}}\left\|v_{m}\right\|^{2} .
\end{aligned}
$$

To prove Lemma 4.3 proper, it remains to apply the above argument to the functions $u_{1}, \ldots, u_{p_{1}+r_{1}}$ and mappings $\mathcal{L}_{m, 1}:=\mathbb{A}_{p_{1}, m-p_{1}-r_{1}}^{n}$, which are coercive due to Assumption 4.1, whereas the other mappings (whose definition involves the matrices $\left.\widetilde{A}_{m, l}^{n}\right)$ are continuous by the boundedness hypothesis on the matrices $A_{\ell}^{n}$ in Assumption 4.3. The reader can check that all bounds are uniform with respect to $n \in \mathbb{N}$.

For $(f, g)=0$ and $F$ arbitrary, we proceed as for $d=1$ and choose $\gamma$ large enough to deal with the correcting term in Gårding's inequality. We then obtain the following two lemmas that we will not prove.

Lemma 4.4. Let Assumptions 4.1, 4.2 and 4.3 be satisfied. Then there exist $C>0$ and $\bar{\gamma}>0$ such that for all $\gamma \geq \bar{\gamma}$ and all $\Delta t \in] 0,1]$, the solution $V$ to (45) with 
$(f, g)=0$ satisfies

$$
\begin{gathered}
\sup _{n \geq 1} \mathrm{e}^{-2 \gamma n \Delta t}\left\|V^{n}\right\|_{1,+\infty}^{2}+\frac{\gamma}{\gamma \Delta t+1} \sum_{n \geq 1} \Delta t \mathrm{e}^{-2 \gamma n \Delta t}\left\|V^{n}\right\|_{1,+\infty}^{2} \\
+\mathrm{e}^{-2 \gamma \Delta t} \sum_{n \geq 1} \Delta t \mathrm{e}^{-2 \gamma n \Delta t} \sum_{j_{1}=1}^{p_{1}} \frac{\left\|V^{n}\right\|_{j_{1}}^{2}}{\Delta x_{1}} \\
\leq C \frac{\gamma \Delta t+1}{\gamma} \sum_{n \geq 0} \Delta t \mathrm{e}^{-2 \gamma(n+1) \Delta t}\left\|F^{n}\right\|_{1,+\infty}^{2} .
\end{gathered}
$$

Lemma 4.5. Let Assumptions 4.1, 4.2 and 4.3 be satisfied. Then there exist $C>0$ and $\bar{\gamma}>0$ such that for all $\gamma \geq \bar{\gamma}$ and all $\Delta t \in] 0,1]$ verifying $\gamma \Delta t \geq 1$, the solution $V$ to (45) with $(f, g)=0$ satisfies

$$
\sum_{n \geq 1} \Delta t \mathrm{e}^{-2 \gamma n \Delta t} \sum_{j_{1}=1}^{p_{1}} \frac{\left\|V^{n}\right\|_{j_{1}}^{2}}{\Delta x_{1}} \leq C \frac{\gamma \Delta t+1}{\gamma} \sum_{n \geq 0} \Delta t \mathrm{e}^{-2 \gamma(n+1) \Delta t}\left\|F^{n}\right\|_{1,+\infty}^{2} .
$$

The combination of Lemmas 4.2, 4.4 and 4.5 yields the corollary:

Corollary 4.1. Let Assumptions 4.1, 4.2 and 4.3 be satisfied. Then there exist $C>0$ and $\bar{\gamma}>0$ such that for all $\gamma \geq \bar{\gamma}$ and all $\Delta t \in] 0,1]$, the solution $V$ to (45) satisfies the estimate

$$
\begin{gathered}
\sup _{n \geq 0} \mathrm{e}^{-2 \gamma n \Delta t}\left\|V^{n}\right\|_{1-r_{1},+\infty}^{2}+\frac{\gamma}{\gamma \Delta t+1} \sum_{n \geq 0} \Delta t \mathrm{e}^{-2 \gamma n \Delta t}\left\|V^{n}\right\|_{1-r_{1},+\infty}^{2} \\
+\sum_{n \geq 0} \Delta t \mathrm{e}^{-2 \gamma n \Delta t} \sum_{j_{1}=1-r_{1}}^{p_{1}} \frac{\left\|V^{n}\right\|_{j_{1}}^{2}}{\Delta x_{1}} \leq C\left\{\|f\|_{1-r_{1},+\infty}^{2}\right. \\
\left.+\frac{\gamma \Delta t+1}{\gamma} \sum_{n \geq 0} \Delta t \mathrm{e}^{-2 \gamma(n+1) \Delta t}\left\|F^{n}\right\|_{1,+\infty}^{2}+\sum_{n \geq 1} \Delta t \mathrm{e}^{-2 \gamma n \Delta t} \sum_{j_{1}=1-r_{1}}^{0} \frac{\left\|g^{n}\right\|_{j_{1}}^{2}}{\Delta x_{1}}\right\} .
\end{gathered}
$$

We are in a position to conclude the proof of Theorem 4.2 .

End of the proof of Theorem 4.2. From now on, we consider the case $q_{1} \geq p_{1}$ since for $q_{1}<p_{1}$, Corollary 4.1 already gives the result of Theorem 4.2, Once again, the proof of (46) is slightly different according to the value of $p_{1}$. For $p_{1} \geq 1$, we proceed along the lines of the proof of Theorem 2.2, and we only treat the case $p_{1}=0$ here.

For all $n \geq 1, m=1, \ldots, q_{1}+1$ and $\ell_{1}=-r_{1}, \ldots, 0$, we set

$$
\begin{aligned}
\mathcal{A}_{\ell_{1}, m}^{n}: \quad L^{2}\left(\left[m \Delta x_{1},(m+1) \Delta x_{1}\left[\times \mathbb{R}^{d-1}\right)\right.\right. & \rightarrow L^{2}\left(\left[m \Delta x_{1},(m+1) \Delta x_{1}\left[\times \mathbb{R}^{d-1}\right)\right.\right. \\
v(x) & \mapsto \sum_{\ell^{\prime}=-r^{\prime}}^{p^{\prime}} A_{\ell_{1}, \ell^{\prime}}^{n}(x) T_{\Delta x^{\prime}}^{\prime}{ }^{\ell^{\prime}} v(x) .
\end{aligned}
$$

Assumption 4.1 with $p_{1}=0$ implies that there exists $\varepsilon_{0}>0$ and norms $\|\cdot\|_{H_{m}^{n}}$ equivalent to $\|\cdot\|_{m}$ in $L^{2}\left(\left[m \Delta x_{1},(m+1) \Delta x_{1}\left[\times \mathbb{R}^{d-1}\right)\right.\right.$, such that

$$
\forall v \in L^{2}\left(\left[0, \Delta x_{1}\left[\times \mathbb{R}^{d-1}\right), \quad\left\|\mathcal{A}_{0, m}^{n} v\right\|_{H_{m}^{n}} \leq \sqrt{1-2 \varepsilon_{0}}\|v\|_{H_{m}^{n}},\right.\right.
$$

for all $n \geq 1$ and $m=1, \ldots, q_{1}+1$. In addition, $\|\cdot\|_{H_{m}^{n}}$ is a Hermitian norm and the constants of equivalence with $\|\cdot\|_{m}$ do not depend on $n \geq 1$ and $m=$ 
$1, \ldots, q_{1}+1$. This result is standard in the case of matrices. The analogue for operators can be found in [5]. As for the case $d=1$, we start from the relation: for all $x \in\left[\Delta x_{1}, 2 \Delta x_{1}\left[\times \mathbb{R}^{d-1}\right.\right.$,

$$
\begin{aligned}
V^{n+1}(x) & =\mathcal{A}_{0,1}^{n} V^{n}(x)+\sum_{\ell_{1}=-r_{1}}^{-1} \mathcal{A}_{\ell_{1}, 1}^{n} T_{\Delta x_{1}}^{\ell_{1}} V^{n}(x)+\Delta t F^{n}(x) \\
& =\mathcal{A}_{0,1}^{n} V^{n}(x)+\underbrace{\sum_{\ell_{1}=-r_{1}}^{-1} \mathcal{A}_{\ell_{1}, 1} T_{\Delta x_{1}}^{\ell_{1}} g^{n}(x)+\Delta t F^{n}(x)}_{=: X^{n}(x)}
\end{aligned}
$$

Note that for $\ell_{1}=-r_{1}, \ldots,-1, T_{\Delta x_{1}}{ }^{\ell_{1}} g^{n}(x)$ is well defined for all $x$ satisfying $x_{1} \in\left[\Delta x_{1}, 2 \Delta x_{1}\left[\times \mathbb{R}^{d-1}\right.\right.$. We then derive

$$
\begin{aligned}
\left\|V^{n+1}\right\|_{H_{1}^{n}}^{2} & =\left\|\mathcal{A}_{0,1}^{n} V^{n}\right\|_{H_{1}^{n}}^{2}+2\left\langle\mathcal{A}_{0,1}^{n} V^{n} ; X^{n}\right\rangle_{H_{1}^{n}}+\left\|X^{n}\right\|_{H_{1}^{n}}^{2} \\
& \leq\left(1-2 \varepsilon_{0}\right)\left\|V^{n}\right\|_{H_{1}^{n}}^{2}+2\left\langle\mathcal{A}_{0,1}^{n} V^{n} ; X^{n}\right\rangle_{H_{1}^{n}}+\left\|X^{n}\right\|_{H_{1}^{n}}^{2} \\
& \leq\left(1-\varepsilon_{0}\right)\left\|V^{n}\right\|_{H_{1}^{n}}^{2}+\left(1+\varepsilon_{0}^{-1}\right)\left\|X^{n}\right\|_{H_{1}^{n}}^{2} .
\end{aligned}
$$

Using the definition of $X^{n}$ and the equivalence of the norms, the latter inequality gives

$$
\left\|V^{n+1}\right\|_{H_{1}^{n}}^{2}-\left\|V^{n}\right\|_{H_{1}^{n}}^{2}+\varepsilon_{0}\left\|V^{n}\right\|_{H_{1}^{n}}^{2} \leq C\left(\Delta t^{2}\left\|F^{n}\right\|_{1,+\infty}^{2}+\sum_{j_{1}=1-r_{1}}^{0}\left\|g^{n}\right\|_{j_{1}}^{2}\right) .
$$

Using the same summation process as earlier, we obtain

$$
\begin{gathered}
\left\{\left(1-\mathrm{e}^{-2 \gamma \Delta t}\right)+\varepsilon_{0} \mathrm{e}^{-2 \gamma \Delta t}\right\} \sum_{n \geq 0} \Delta t \mathrm{e}^{-2 \gamma n \Delta t} \frac{\left\|V^{n}\right\|_{H_{1}^{n}}^{2}}{\Delta x_{1}} \leq C\left\{\|f\|_{1-r_{1},+\infty}^{2}\right. \\
\left.+\frac{\gamma \Delta t+1}{\gamma} \sum_{n \geq 0} \Delta t \mathrm{e}^{-2 \gamma(n+1) \Delta t}\left\|F^{n}\right\|_{1,+\infty}^{2}+\sum_{n \geq 1} \Delta t \mathrm{e}^{-2 \gamma n \Delta t} \sum_{j_{1}=1-r_{1}}^{0} \frac{\left\|g^{n}\right\|_{j_{1}}^{2}}{\Delta x_{1}}\right\} .
\end{gathered}
$$

Since the norms $\|\cdot\|_{H_{1}^{n}}$ and $\|\cdot\|_{1}$ are equivalent, uniformly in $n$, this becomes

$$
\begin{aligned}
& \sum_{n \geq 0} \Delta t \mathrm{e}^{-2 \gamma n \Delta t} \frac{\left\|V^{n}\right\|^{2}}{\Delta x_{1}} \leq C\left\{\|f\|_{1-r_{1},+\infty}^{2}\right. \\
& \left.+\frac{\gamma \Delta t+1}{\gamma} \sum_{n \geq 0} \Delta t \mathrm{e}^{-2 \gamma(n+1) \Delta t}\left\|F^{n}\right\|_{1,+\infty}^{2}+\sum_{n \geq 1} \Delta t \mathrm{e}^{-2 \gamma n \Delta t} \sum_{j_{1}=1-r_{1}}^{0} \frac{\left\|g^{n}\right\|_{j_{1}}^{2}}{\Delta x_{1}}\right\},
\end{aligned}
$$

with a constant $C$ that does not depend on $\gamma$ nor on $\Delta t$. The proof of (46) follows from an induction argument where we apply the above method to recover the estimate for the trace of $V^{n}$ on $\left[2 \Delta x_{1},\left(q_{1}+2\right) \Delta x_{1}\left[\times \mathbb{R}^{d-1}\right.\right.$. 
4.3. Proof of Theorem 4.1. We follow once more the proof in the one-dimensional case, and decompose the solution $U$ to (42) as $U=V+W$, where $V$ satisfies $(57)$

$$
\begin{cases}V^{n+1}(x)=Q^{n}(x) V^{n}(x)+\Delta t F^{n}(x), & x \in\left[\Delta x_{1},+\infty\left[\times \mathbb{R}^{d-1}, \quad n \geq 0,\right.\right. \\ V^{n+1}(x)=g^{n+1}(x), & x \in\left[\left(1-r_{1}\right) \Delta x_{1}, \Delta x_{1}\left[\times \mathbb{R}^{d-1}, \quad n \geq 0,\right.\right. \\ V^{0}(x)=f(x), & x \in\left[\left(1-r_{1}\right) \Delta x_{1},+\infty\left[\times \mathbb{R}^{d-1},\right.\right.\end{cases}
$$

and $W$ satisfies

(58)

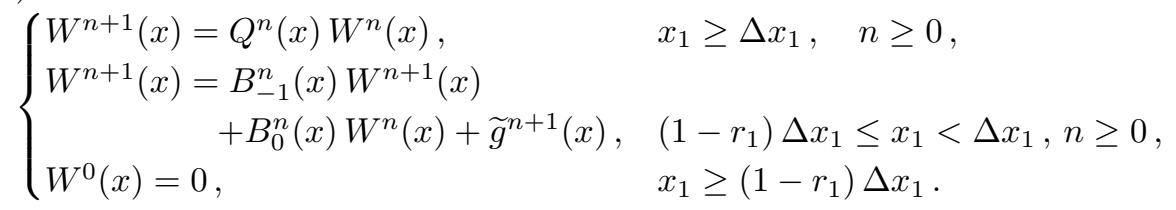

The source term $\widetilde{g}$ in (58) is now defined by

$\widetilde{g}^{n}(x):=B_{-1}^{n}(x) V^{n}(x)+B_{0}^{n}(x) V^{n-1}(x), \quad\left(1-r_{1}\right) \Delta x_{1} \leq x_{1}<\Delta x_{1}, \quad \forall n \geq 1$.

The estimate of $V$ is given by Theorem 4.2. Since the discretization (42) is strongly stable in the sense of Definition 4.1, we already know that $W$ satisfies

$$
\begin{aligned}
\frac{\gamma}{\gamma \Delta t+1} \sum_{n \geq 0} \Delta t \mathrm{e}^{-2 \gamma n \Delta t}\left\|W^{n}\right\|_{1-r_{1},+\infty}^{2} & +\sum_{n \geq 0} \Delta t \mathrm{e}^{-2 \gamma n \Delta t} \sum_{j_{1}=1-r_{1}}^{0} \frac{\left\|W^{n}\right\|_{j_{1}}^{2}}{\Delta x_{1}} \\
& \leq C \sum_{n \geq 1} \Delta t \mathrm{e}^{-2 \gamma n \Delta t} \sum_{j_{1}=1-r_{1}}^{0} \frac{\left\|\widetilde{g}^{n}\right\|_{j_{1}}^{2}}{\Delta x_{1}}
\end{aligned}
$$

for $\gamma$ large enough. The estimate of $\widetilde{g}^{n}$ follows from the definition (59), and (46). In the end, we obtain

$$
\begin{gathered}
\frac{\gamma}{\gamma \Delta t+1} \sum_{n \geq 0} \Delta t \mathrm{e}^{-2 \gamma n \Delta t}\left\|W^{n}\right\|_{1-r_{1},+\infty}^{2}+\sum_{n \geq 0} \Delta t \mathrm{e}^{-2 \gamma n \Delta t} \sum_{j_{1}=1-r_{1}}^{0} \frac{\left\|W^{n}\right\|_{j_{1}}^{2}}{\Delta x_{1}} \\
\leq C\left\{\|f\|_{1-r_{1},+\infty}^{2}+\frac{\gamma \Delta t+1}{\gamma} \sum_{n \geq 0} \Delta t \mathrm{e}^{-2 \gamma(n+1) \Delta t}\left\|F^{n}\right\|_{1,+\infty}^{2}\right. \\
\left.+\sum_{n \geq 1} \Delta t \mathrm{e}^{-2 \gamma n \Delta t} \sum_{j_{1}=1-r_{1}}^{0} \frac{\left\|\widetilde{g}^{n}\right\|_{j_{1}}^{2}}{\Delta x_{1}}\right\} .
\end{gathered}
$$

Provided we control the $\ell_{n}^{\infty}\left(L_{x}^{2}\right)$ norm of $W$, Theorem 4.1 is a consequence of the combination of (60) for $W$ and (46) for $V$. This control is given by

Lemma 4.6. Let Assumptions 4.2 and 4.3 be satisfied, and assume that the discretization (42) is strongly stable in the sense of Definition 4.1. Then there exist $C>0$ and $\bar{\gamma}>0$ independent of the data $\widetilde{g}$, such that for all $\gamma \geq \bar{\gamma}$ and all $\Delta t \in] 0,1]$, the solution $W$ to (58) satisfies

$$
\sup _{n \geq 0} \mathrm{e}^{-2 \gamma n \Delta t}\left\|W^{n}\right\|_{1-r,+\infty}^{2} \leq C \sum_{n \geq 1} \Delta t \mathrm{e}^{-2 \gamma n \Delta t} \sum_{j_{1}=1-r_{1}}^{0} \frac{\left\|\widetilde{g}^{n}\right\|_{j_{1}}^{2}}{\Delta x_{1}} .
$$


The proof of Lemma 4.6 is similar to the proof of Lemma 2.5 provided the same adaptations as above pass from one-dimensional to multidimensional problems and from constant to variable coefficients. We leave the details to the interested reader.

\section{EXAMPLES AND COMMENTS}

5.1. Examples and comments in one space dimension. For one-dimensional problems, the matrices $A_{\ell}$ in the finite difference operator $Q$ are usually polynomials of the matrix $\lambda A$ where $A$ is the matrix of the hyperbolic operator in (1) and $\lambda=$ $\Delta t / \Delta x$ is the CFL parameter. If we assume furthermore that $A$ is real symmetric, then there exists an orthogonal matrix that diagonalizes simultaneously all the matrices $A_{\ell}$. In this case, Assumption 2.2 is exactly equivalent to the $\ell^{2}$-stability of the finite difference approximation, which is itself equivalent to the well-known von Neumann condition (see [3, Chapter 5])

$$
\forall \kappa \in \mathbb{S}^{1}, \quad \rho\left(\sum_{\ell=-r}^{p} \kappa^{\ell} A_{\ell}\right) \leq 1 .
$$

We recall that $\rho$ denotes the spectral radius of a square complex matrix. Assumption 2.2 is therefore very reasonable and not restrictive in one space dimension. Assumption 2.1 is also satisfied in numerous situations and is rather easy to check.

We now make a few comments on the proof of Theorem 2.2. The proof in the case $F=0$ follows from Lemma 2.1 and is done in a single way, whatever the values of $\gamma$ and $\Delta t$ are. However, we have seen that the case $F \neq 0$ requires two different approaches depending on the value of $\gamma \Delta t$. Here, we report on a simple numerical test which shows that the two regimes $\gamma \Delta t \leq 1$ and $\gamma \Delta t \geq 1$ are different when $F \neq 0$. We consider the scalar transport equation

$$
\begin{cases}\partial_{t} u-\partial_{x} u=F(t, x), & (t, x) \in \mathbb{R}^{+} \times \mathbb{R}^{+}, \\ u(0, x)=f(x), & x \in \mathbb{R}^{+},\end{cases}
$$

for which no boundary condition is required. We discretize the equation with the Lax-Friedrichs scheme and a homogeneous Dirichlet boundary condition 4

$$
\left\{\begin{array}{rlrl}
v_{j}^{n+1}=\left(v_{j-1}^{n}+v_{j+1}^{n}\right) / 2+\lambda\left(v_{j+1}^{n}-v_{j-1}^{n}\right) / 2 & & \\
& +\Delta t F(n \Delta t, j \Delta x), & & j \geq 1, \quad n \geq 0, \\
v_{0}^{n+1}=0, & & n \geq 0, \\
v_{j}^{0}=f(j \Delta x), & & j \geq 0,
\end{array}\right.
$$

or with the Lax-Wendroff scheme and a homogeneous Dirichlet boundary condition

$$
\left\{\begin{array}{rlrl}
v_{j}^{n+1}=v_{j}^{n}+\lambda\left(v_{j+1}^{n}-v_{j-1}^{n}\right) / 2 & \\
& +\lambda^{2}\left(v_{j+1}^{n}+v_{j-1}^{n}-2 v_{j}^{n}\right) / 2+\Delta t F(n \Delta t, j \Delta x), & & j \geq 1, n \geq 0, \\
v_{0}^{n+1}=0, & & n \geq 0, \\
v_{j}^{0}=f(j \Delta x), & & j \geq 0 .
\end{array}\right.
$$

The CFL parameter $\lambda$ is 0.9 in both situations, which ensures that Theorem 2.2 holds (Assumptions 2.1 and 2.2 are satisfied).

\footnotetext{
${ }^{4}$ Observe that the homogeneous Dirichlet condition is not consistent in the $L^{\infty}$-norm with the continuous problem for which no boundary condition is required. However, we are concerned here with stability estimates, and consistency is a different issue.
} 

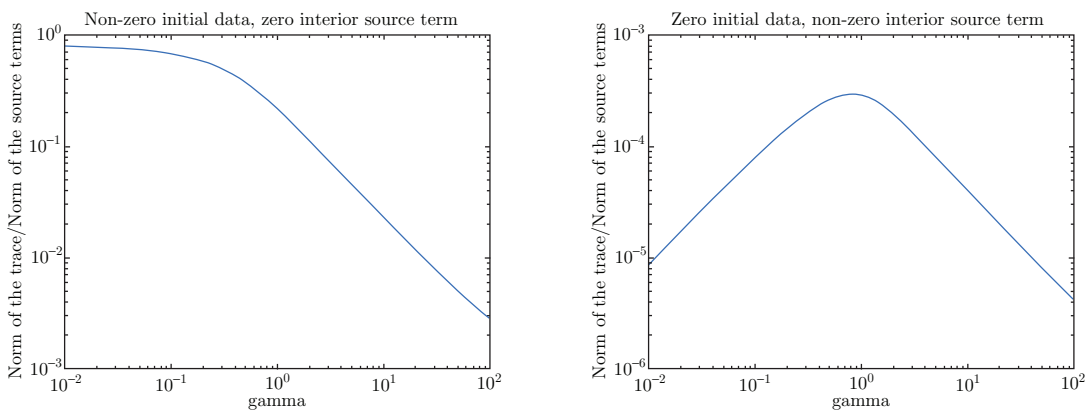

FiguRE 1. Lax-Friedrichs scheme (61): ratio between the norm of the trace and the norm of the source terms. Non-zero initial data and zero interior source term (left). Zero initial data and non-zero interior source term (right).

We plot the the ratio between the norm of the trace

$$
\sum_{n \geq 0} \Delta t \mathrm{e}^{-2 \gamma n \Delta t}\left|v_{1}^{n}\right|^{2}
$$

and the right-hand side of (8) as a function of $\gamma$ in the two following cases:

(1) $f(x)=1$ for $x \in[0,1], F=0$. The simulation is performed on the space interval $[0,2]$ with 1000 grid points. The parameter $\gamma$ ranges from $10^{-2}$ to $10^{2}$.

(2) $f=0, F(t, x)=1$ for $t \geq 0$ and $x \in[0,1]$. The simulation is performed on the space interval $[0,2]$ with 1000 grid points. The parameter $\gamma$ ranges from $10^{-2}$ to $10^{2}$.

The results are plotted in Figure 1 for the Lax-Friedrichs scheme (61), and in Figure 2 for the Lax-Wendroff scheme (62). The observation is the following: the ratio between the norm of the trace and the norm of the source term depends monotonically on $\gamma$ when $f \neq 0$ and $F=0$, while it does not depend monotonically on $\gamma$ when $f=0$ and $F \neq 0$. This seems to indicate that in the case $F \neq 0$, the estimate (8) for small values of $\gamma$ does not follow from the same arguments as for large values of $\gamma$. This is the reason why we believe that Lemmas 2.3 and 2.4 are both useful to obtain a stability estimate with a constant that is independent of $\gamma$.

5.2. Examples and comments in several space dimensions. Let us first comment on our multidimensional version of the Golberg-Tadmor Lemma. In one dimension, the commutation assumption by Golberg and Tadmor is very natural, and essentially, it is "equivalent" to Assumption 2.2. The original work by Golberg and Tadmor [2] also covers the multidimensional case provided the matrices $\left\{A_{\ell}\right\}$ in (32) commute. This assumption is very restrictive for $d>1$ and amounts more or less to consider $d$ uncoupled scalar equations in (29). Assumption 3.2. however, can hold independently of the fact that the matrices $\left\{A_{\ell}\right\}$ do commute or not. Theorem 3.2 is therefore a true generalization of the Golberg-Tadmor Lemma.

In the remaining part of this paragraph, we consider $d=2$ and give several examples of discretizations to which Theorem 3.1 applies. 

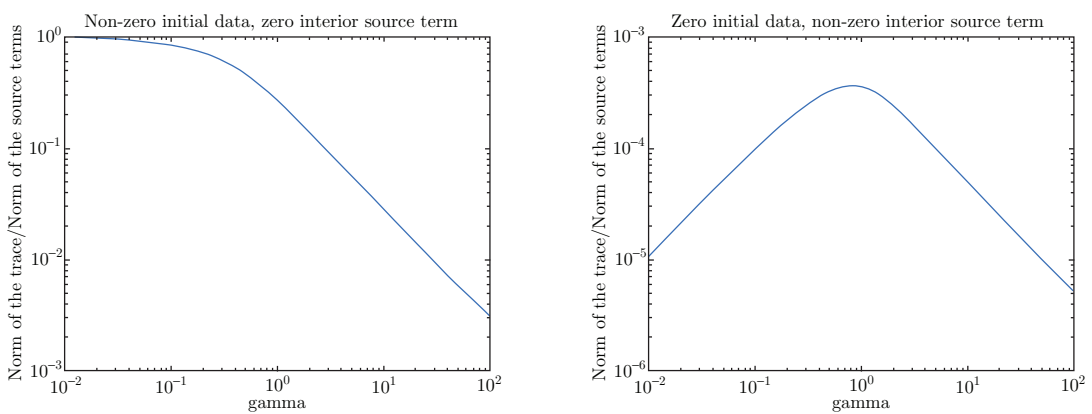

FiguRE 2. Lax-Wendroff scheme (62): ratio between the norm of the trace and the norm of the source terms. Non-zero initial data and zero interior source term (left). Zero initial data and non-zero interior source term (right).

We consider the following two-dimensional problem:

$$
\begin{cases}\partial_{t} u+A_{1} \partial_{x_{1}} u+A_{2} \partial_{x_{2}} u=F(t, x), & (t, x) \in \mathbb{R}^{+} \times \mathbb{R}_{+}^{2}, \\ B u\left(t, 0, x^{\prime}\right)=g\left(t, x^{\prime}\right), & t \in \mathbb{R}^{+}, x^{\prime} \in \mathbb{R}, \\ u(0, x)=f(x), & x \in \mathbb{R}_{+}^{2},\end{cases}
$$

where $A_{1}, A_{2}$ and $B$ are matrices, and $A_{1}$ and $A_{2}$ are real symmetric. We assume that $f \in L^{2}\left(\mathbb{R}_{+}^{2}\right)$, and that there exists $\gamma>0$ such that $g \in \mathrm{e}^{\gamma t} L^{2}\left(\mathbb{R}^{+} ; L^{2}(\mathbb{R})\right)$, and $F \in \mathrm{e}^{\gamma t} L^{2}\left(\mathbb{R}^{+} ; L^{2}\left(\mathbb{R}^{2}\right)\right)$. We let $f_{j_{1}, j_{2}}, F_{j_{1}, j_{2}}^{n}$ and $g_{j_{1}, j_{2}}^{n}$ be discrete approximations of $f, F$ and $g$ at time $n \Delta t$, and points $\left(j_{1} \Delta x_{1}, j_{2} \Delta x_{2}\right)$, where $\Delta t, \Delta x_{1}$ and $\Delta x_{2}$ are the time and space steps related through the fixed CFL ratios $\lambda_{1}=\Delta x_{1} / \Delta t$ and $\lambda_{2}=\Delta x_{2} / \Delta t$. As before, $U_{j_{1}, j_{2}}^{n}$ denotes the approximation of the solution $u$ to (63) at time $n \Delta t$, and points $\left(j_{1} \Delta x_{1}, j_{2} \Delta x_{2}\right)$. As for the one-dimensional examples (61) and (62), we address stability issues, not consistency, and we replace the boundary condition in (63) by Dirichlet boundary conditions for the numerical scheme.

5.2.1. The Lax-Friedrichs scheme. The Lax-Friedrichs scheme with Dirichlet boundary condition reads

$$
\left\{\begin{array}{lll}
U_{j_{1}, j_{2}}^{n+1}=Q_{L F} U_{j_{1}, j_{2}}^{n}+\Delta t F_{j_{1}, j_{2}}^{n}, & & \text { for } j_{1} \geq 1, j_{2} \in \mathbb{Z}, \quad n \geq 0, \\
U_{j_{1}, j_{2}}^{n+1}=g_{j_{1}, j_{2}}^{n+1}, & \text { for } j_{1}=0, j_{2} \in \mathbb{Z}, \quad n \geq 0, \\
U_{j_{1}, j_{2}}^{0}=f_{j_{1}, j_{2}}, & \text { for } j_{1} \geq 0, j_{2} \in \mathbb{Z}, &
\end{array}\right.
$$

with the operator $Q_{L F}$ given by

$$
Q_{L F}:=\frac{1}{4}\left(T_{1}^{-1}+T_{1}+T_{2}^{-1}+T_{2}\right)-\frac{\lambda_{1}}{2} A_{1}\left(T_{1}-T_{1}^{-1}\right)-\frac{\lambda_{2}}{2} A_{2}\left(T_{2}-T_{2}^{-1}\right) .
$$

In particular, it is of the form (31) with $p_{1}=p_{2}=r_{1}=r_{2}=1, q_{1}=q_{2}=0$, and obvious definitions for the matrices $A_{j_{1}, j_{2}}$. Assumptions 3.1 and 3.2 are translated in terms of admissible zones for the CFL parameters $\left(\lambda_{1}, \lambda_{2}\right)$.

Let us begin with Assumption 3.1. In this case, $p_{1}=1$, and the mapping

$$
\mathbb{A}_{1}(z): w \in \ell^{2}(\mathbb{Z}) \mapsto-\frac{1}{z}\left(\frac{1}{4} w-\frac{\lambda_{1}}{2} A_{1} w\right)=-\frac{1}{4 z}\left(I-2 \lambda_{1} A_{1}\right) w
$$


has to be coercive on $\ell^{2}(\mathbb{Z})$ for all $|z| \geq 1$. Since $A_{1}$ is diagonalizable, a sufficient condition for Assumption 3.1 to be satisfied is

$$
\lambda_{1} \rho\left(A_{1}\right)<1 / 2 .
$$

Assumption 3.2 is particularly simple in this case, because the symbol of the discretized operator $Q_{L F}$ is a normal matrix. Hence it is diagonalizable in an orthonormal basis and its Hermitian norm coincides with its spectral radius. We refer to [15] for the following sufficient condition for Assumption 3.2 to hold:

$$
\forall \theta \in[0,2 \pi], \quad \rho\left(\lambda_{1} \cos \theta A_{1}+\lambda_{2} \sin \theta A_{2}\right) \leq \frac{1}{\sqrt{2}} .
$$

As shown in [14, the latter condition holds provided that we have 5

$$
\lambda_{1}^{2} A_{1}^{2}+\lambda_{2}^{2} A_{2} \leq \frac{1}{2} I .
$$

Provided conditions (65) and (66) hold, Theorem 3.2 shows that the solution to (64) satisfies estimate (41).

5.2.2. The Modified Lax-Wendroff scheme I. Let us now address a variant of the Lax-Wendroff scheme introduced by Wendroff in [16] whose $\ell^{2}$-stability has been further studied by Vaillancourt in [15]. With the notation of Section 3, the finite difference operator reads

$$
\begin{aligned}
Q_{L W 1}:= & I-\frac{1}{8}\left(\lambda_{1} A_{1}\left(T_{1}-T_{1}^{-1}\right)+\lambda_{2} A_{2}\left(T_{2}-T_{2}^{-1}\right)\right) \\
& \left(T_{1}+T_{1}^{-1}+T_{2}+T_{2}^{-1}-\lambda_{1} A_{1}\left(T_{1}-T_{1}^{-1}\right)-\lambda_{2} A_{2}\left(T_{2}-T_{2}^{-1}\right)\right) .
\end{aligned}
$$

For this scheme, $p_{1}=2$ and for all $j_{2} \in \mathbb{Z}$,

$$
A_{2, j_{2}}=-\frac{1}{8} \lambda_{1} A_{1}\left(I-\lambda_{1} A_{1}\right) \delta_{0 j_{2}} .
$$

We first determine the CFL parameters $\left(\lambda_{1}, \lambda_{2}\right)$ for which Assumption 3.2 is satisfied. The symbol of the discretized operator $Q_{L W 1}$ is given by

$$
G(\xi):=I-i J(\xi)\left(c(\xi) I-i \frac{J(\xi)}{2}\right)=I-\frac{J(\xi)^{2}}{2}-i c(\xi) J(\xi),
$$

where $J(\xi):=\sin \xi_{1} \lambda_{1} A_{1}+\sin \xi_{2} \lambda_{2} A_{2}$, and $c(\xi):=\left(\cos \xi_{1}+\cos \xi_{2}\right) / 2$. In particular, $G(\xi)$ is a normal matrix. Hence, $G(\xi)$ is diagonalizable in an orthonormal basis and the scheme is $\ell^{2}$-stable if and only if Assumption 3.2 is satisfied. This stability condition is equivalent to the fulfillment of the von Neumann condition, which reads $G(\xi) G^{*}(\xi) \leq I$, that is,

$$
J(\xi)^{2} \leq 4(1-c(\xi)) I .
$$

Following [15], one notes that

$$
1-c(\xi)^{2}=\frac{1}{2}\left(\sin ^{2} \xi_{1}+\sin ^{2} \xi_{2}\right)+\frac{1}{4}\left(\cos \xi_{1}-\cos \xi_{2}\right)^{2} .
$$

Hence, Assumption 3.2 holds, provided

$$
\left(\sin \xi_{1} \lambda_{1} A_{1}+\sin \xi_{2} \lambda_{2} A_{2}\right)^{2} \leq 2\left(\sin ^{2} \xi_{1}+\sin ^{2} \xi_{2}\right) I .
$$

\footnotetext{
${ }^{5}$ Condition (66) is sufficient for stability, but it is also necessary when the matrices $A_{1}$ and $A_{2}$ commute.
} 
This condition is equivalent to

$$
\forall \theta \in[0,2 \pi], \quad\left(\lambda_{1} \cos \theta A_{1}+\lambda_{2} \sin \theta A_{2}\right)^{2} \leq 2 I .
$$

Therefore, $Q_{L W 1}$ satisfies Assumption 3.2 provided

$$
\lambda_{1}^{2} A_{1}^{2}+\lambda_{2}^{2} A_{2}^{2} \leq 2 I .
$$

Let us now turn to Assumption 3.1. In this case, for all $z \in \mathbb{C}^{*}$, we have

$$
\mathbb{A}_{2}(z): w \mapsto \frac{1}{8 z} \lambda_{1} A_{1}\left(I-\lambda_{1} A_{1}\right) w,
$$

and $\mathbb{A}_{2}(z)$ is nothing but a simple multiplication. The mapping $\mathbb{A}_{2}(z)$ is coercive if

$$
\operatorname{det} A_{1} \neq 0 \quad \text { and } \quad \lambda_{1} \rho_{1}\left(A_{1}\right)<1 .
$$

Consequently, Theorem 3.2 shows stability for the modified Lax-Wendroff scheme (67) with Dirichlet boundary conditions provided (68) and (69) hold.

5.2.3. The Modified Lax-Wendroff scheme II. Our last example is another modification of the Lax-Wendroff scheme introduced by Lax and Wendroff in [9]. The discretized operator is now

$$
\begin{aligned}
Q_{L W 2}:=I-\frac{1}{2}\left(\lambda_{1} A_{1}\right. & \left.\left(T_{1}-T_{1}^{-1}\right)+\lambda_{2} A_{2}\left(T_{2}-T_{2}^{-1}\right)\right) \\
& +\frac{1}{8}\left(\lambda_{1} A_{1}\left(T_{1}-T_{1}^{-1}\right)+\lambda_{2} A_{2}\left(T_{2}-T_{2}^{-1}\right)\right)^{2}-L,
\end{aligned}
$$

where

$L:=\frac{1}{8}\left(T_{1}+T_{1}^{-1}+T_{2}+T_{2}^{-1}-4 I\right)\left(\lambda_{1}^{2} A_{1}^{2}\left(T_{1}+T_{1}^{-1}-2 I\right)+\lambda_{2}^{2} A_{2}^{2}\left(T_{2}+T_{2}^{-1}-2 I\right)\right)$.

Although it may seem at first glance that the scheme involves 5 points in each direction, there are cancellations for the terms $T_{j}^{2}, T_{j}^{-2}$ and we have $r_{1}=r_{2}=p_{1}=$ $p_{2}=1$.

The $\ell^{2}$-stablity of $Q_{L W 2}$ has been characterized by Lax and Wendroff in [9]; see also Turkel [14. However, there is a small gap between the general concept of $\ell^{2}$-stablity for which the operators $Q_{L W 2}^{n}$ are bounded uniformly in $n \in \mathbb{N}$, and Assumption 3.2 where we require the norm of $Q_{L W 2}$ to be no larger than 1. The latter property is called strong $\ell^{2}$-stability by Tadmor in [13. The results of 9 ] and [13] show that the discretized operator $Q_{L W 2}$ in (70) is $\ell^{2}$-stable if

$$
\lambda_{1}^{2} A_{1}^{2}+\lambda_{2}^{2} A_{2}^{2} \leq \frac{1}{2} I
$$

while Assumption 3.2 is satisfied under the slightly more restrictive condition

$$
\lambda_{1}^{4} A_{1}^{4}+\lambda_{2}^{4} A_{2}^{4} \leq \frac{1}{8} I .
$$

Let us now address Assumption 3.1. Unlike the previous examples, $\mathbb{A}_{1}(z)$ is no longer a multiplication. Here, for all $z \in \mathbb{C} \backslash\{0\}$, we have

$$
\begin{array}{r}
\mathbb{A}_{1}(z): w \in \ell^{2}(\mathbb{Z}) \mapsto \frac{1}{2 z}\left[\lambda_{1} A_{1}+\lambda_{1}^{2} A_{1}^{2}+\frac{\lambda_{1} \lambda_{2}}{2}\left(A_{1} A_{2}+A_{2} A_{1}\right)\left(T_{2}-T_{2}^{-1}\right)\right. \\
\left.+\frac{1}{4}\left(\lambda_{1}^{2} A_{1}^{2}+\lambda_{2}^{2} A_{2}^{2}\right)\left(T_{2}+T_{2}^{-1}-2 I\right)\right] w,
\end{array}
$$


and Assumption 3.1 is satisfied if and only if $\mathbb{A}_{1}(1 / 2)$ is coercive. The symbol of $\mathbb{A}_{1}(1 / 2)$ is given by

$$
\widehat{\mathcal{A}}\left(\xi_{2}\right):=\lambda_{1} A_{1}\left(I+\lambda_{1} A_{1}\right)-\left(\lambda_{1}^{2} A_{1}^{2}+\lambda_{2} A_{2}^{2}\right) \sin ^{2} \frac{\xi_{2}}{2}+i \frac{\lambda_{1} \lambda_{2}}{2}\left(A_{1} A_{2}+A_{2} A_{1}\right) \sin \xi_{2} .
$$

Using Plancherel's Theorem, it is rather easy to prove that the operator $\mathbb{A}_{1}(1 / 2)$ is coercive if and only if its symbol $\widehat{\mathcal{A}}\left(\xi_{2}\right)$ is invertible for all $\xi_{2} \in \mathbb{R}$.

Let $C_{0}>0$ denote an arbitrary constant. Then for $\lambda_{2} \leq C_{0} \lambda_{1}$, we have

$$
\widehat{\mathcal{A}}\left(\xi_{2}\right)=\lambda_{1} A_{1}+O\left(\lambda_{1}^{2}\right)
$$

uniformly in $\xi_{2} \in \mathbb{R}$. Hence, Assumption 3.1 holds if $\operatorname{det} A_{1} \neq 0$ and $\lambda_{1}$ is small enough (the second CFL parameter $\lambda_{2}$ is subject to the restriction $\lambda_{2} \leq C_{0} \lambda_{1}$ ). In this case, Theorem 3.2 shows the stability of the scheme (70) with Dirichlet boundary conditions and non-zero initial data.

Let us take a closer look at Assumption 3.1 on one specific example. To this aim, we consider

$$
A_{1}=\left(\begin{array}{cc}
1 & 0 \\
0 & -1
\end{array}\right), \quad A_{2}=\left(\begin{array}{ll}
0 & 1 \\
1 & 0
\end{array}\right)
$$

These matrices satisfy $A_{1}^{2}=A_{2}^{2}=I$ and $A_{1} A_{2}+A_{2} A_{1}=0$, so that the symbol $\widehat{\mathcal{A}}$ above reduces to

$$
\widehat{\mathcal{A}}\left(\xi_{2}\right)=\left(\begin{array}{cc}
\lambda_{1}+\lambda_{1}^{2}-\left(\lambda_{1}^{2}+\lambda_{2}^{2}\right) \sin ^{2} \frac{\xi_{2}}{2} & 0 \\
0 & -\lambda_{1}+\lambda_{1}^{2}-\left(\lambda_{1}^{2}+\lambda_{2}^{2}\right) \sin ^{2} \frac{\xi_{2}}{2}
\end{array}\right) .
$$

Let $X:=\sin ^{2}\left(\xi_{2} / 2\right) \in[0,1]$. The symbol $\widehat{\mathcal{A}}\left(\xi_{2}\right)$ is non-invertible if and only if $\lambda_{1}+\lambda_{2}^{2}-\left(\lambda_{1}^{2}+\lambda_{2}^{2}\right) X=0$, that is,

$$
X=\frac{\lambda_{1}+\lambda_{1}^{2}}{\lambda_{1}^{2}+\lambda_{2}^{2}}
$$

Since $X \in[0,1]$, this may only happen for $\lambda_{1} \leq \lambda_{2}^{2}$. Hence, Assumption 3.1 is satisfied if and only if

$$
\lambda_{2}<\sqrt{\lambda_{1}} .
$$

Theorem 3.2 then shows that the scheme (70) with Dirichlet boundary conditions and general initial data is stable provided (71) and (72) hold. In this case, Assumption 3.1 does have an impact on the stability region, as illustrated on Figure 3. In particular, the condition (72) does not only involve $\lambda_{1}$ but also $\lambda_{2}$. The strong stability region for $Q_{L W 2}$ (Assumption 3.2 satisfied) in the $\left(\lambda_{1}, \lambda_{2}\right)$-plane corresponds to the grey and black disk, whereas the black region corresponds to the fulfillment of both (71) and (72). 


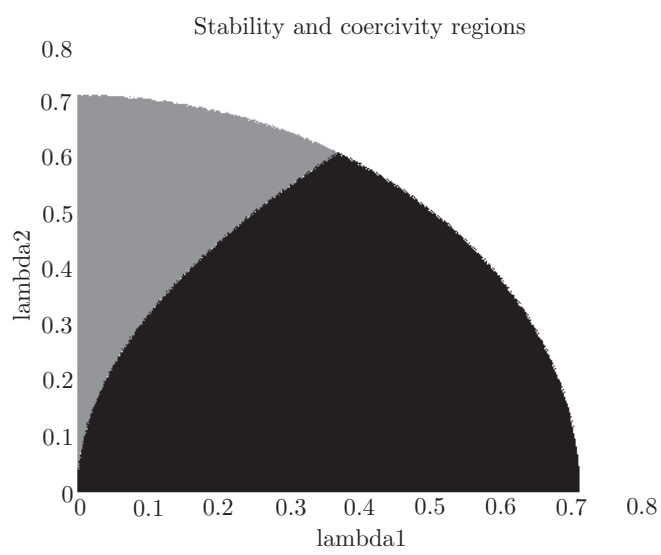

FiguRE 3. Strong stability (grey and black) and coercivity (black) regions for the modified Lax-Wendroff scheme II in the plane $\left(\lambda_{1}, \lambda_{2}\right)$ for the specific example.

\section{REFERENCES}

1. S. Benzoni-Gavage, D. Serre, Multidimensional hyperbolic partial differential equations, Oxford University Press, 2007, First-order systems and applications. MR2284507 (2008k:35002)

2. M. Goldberg, E. Tadmor, Scheme-independent stability criteria for difference approximations of hyperbolic initial-boundary value problems. II, Math. Comp. 36 (1981), no. 154, 603-626. MR.606519 (83f:65142)

3. B. Gustafsson, H.-O. Kreiss, and J. Oliger, Time dependent problems and difference methods, John Wiley \& Sons, 1995. MR 1377057 (97c:65145)

4. B. Gustafsson, H.-O. Kreiss, and A. Sundström, Stability theory of difference approximations for mixed initial boundary value problems. II, Math. Comp. 26 (1972), no. 119, 649-686. MR 0341888 (49:6634)

5. R. D. Holmes, A formula for the spectral radius of an operator, Amer. Math. Month. 75 (1968), no. 2, 163-166. MR0227783 (37:3367)

6. H.-O. Kreiss, Difference approximations for hyperbolic differential equations, Numerical Solution of Partial Differential Equations (Proc. Sympos. Univ. Maryland, 1965), Academic Press, New York, 1966, pp. 51-58. MR0207223 (34:7039)

7. Stability theory for difference approximations of mixed initial boundary value problems. I, Math. Comp. 22 (1968), 703-714. MR0241010(39:2355)

8. _ Initial boundary value problems for hyperbolic systems, Comm. Pure Appl. Math. 23 (1970), 277-298. MR0437941(55:10862)

9. P. D. Lax, B. Wendroff, Difference schemes for hyperbolic equations with high order of accuracy, Comm. Pure Appl. Math. 17 (1964), 381-398. MR0170484 (30:722)

10. P. D. Lax, L. Nirenberg, On stability for difference schemes: A sharp form of Gärding's inequality, Comm. Pure Appl. Math. 19 (1966), 473-492. MR0206534 (34:6352)

11. D. Michelson, Stability theory of difference approximations for multidimensional initialboundary value problems, Math. Comp. 40 (1983), no. 161, 1-45. MR679433 (84d:65068)

12. J. Rauch, $\mathcal{L}^{2}$ is a continuable initial condition for Kreiss' mixed problems, Comm. Pure Appl. Math. 25 (1972), 265-285. MR0298232 (45:7284)

13. E. Tadmor, Complex symmetric matrices with strongly stable iterates, Linear Algebra Appl. 78 (1986), 65-77. MR840168 (87f:15016)

14. E. Turkel, Symmetric hyperbolic difference schemes and matrix problems, Linear Algebra and Appl. 16 (1977), no. 2, 109-129. MR 0464603 (57:4530)

15. R. Vaillancourt, On the stability of Friedrichs' scheme and the modified Lax-Wendroff scheme, Math. Comp. 24 (1970), 767-770. MR0277125 (43:2862) 
16. B. Wendroff, Well-posed problems and stable difference operators, SIAM J. Numer. Anal. 5 (1968), 71-82. MR0223110 (36:6159)

17. L. Wu, The semigroup stability of the difference approximations for initial-boundary value problems, Math. Comp. 64 (1995), no. 209, 71-88. MR1257582 (95c:65170)

CNRS, Université Lille 1 and Team Project Simpaf of inRia lille - Nord Europe, Laboratoire Paul Painlevé (UMR CNRS 8524), BÂtiment M2, Cité Scientifique, 59655 Villeneuve d'AscQ Cedex, France

E-mail address: jean-francois.coulombel@math.univ-lille1.fr

Team Project Simpaf of INRIa lille - Nord Europe, Park Plazza, 40 avenue Halley, 59655 Villeneuve d'Ascq Cedex, France

E-mail address: antoine.gloria@inria.fr 